\title{
Design of Spline-Evolutionary Computing Paradigm for Nonlinear Thin Film Flow Model
}

\author{
Aamir Rizwan ${ }^{1} \cdot$ Iftikhar Ahmad $^{1}$. Muhammad Asif Zahoor Raja ${ }^{2,3} \cdot$ Muhammad Shoaib ${ }^{4}$
}

Received: 2 February 2021 / Accepted: 4 June 2021 / Published online: 2 July 2021

(c) King Fahd University of Petroleum \& Minerals 2021

\begin{abstract}
A novel design of stochastic numerical computing method is introduced for computational fluid dynamics problem governed with nonlinear thin film flow (TFF) system by exploiting the competency of polynomial splines for discretization and optimization with evolutionary computing aided with brilliance of local search. The TFF model of second grade fluid is represented with nonlinear second-order differential system. The aim of the present work is to exploit the cubic spline approach (CSA) to transform the differential equations for TFF model into an equivalent set of nonlinear equations. The approximation in mean squared error sense is introduced for the formulation of cost function for solving the nonlinear system of equations representing TFF model. The optimization of the decision variables of the cost function is carried out with global search efficacy of evolution by genetic algorithms (GAs) integrated with sequential quadratic programming (SQP) for speedy adjustments. The designed spline-evolutionary computing paradigm, CSA-GA-SQP, is evaluated for different scenarios of TFF model by variation of second grade and magnetic parameters, as well as variation in the length of splines. Results endorsed the worth of CSA-GA-SQP solver as an efficient alternative, reliable, stable, and accurate framework for the variants of nonlinear TFF systems on the basis of multiple autonomous executions. The design computing spline paradigm CSA-GA-SQP is a promising alternative numerical solver to be implemented for the solution of stiff nonlinear systems representing the complex scenarios of computational fluid dynamics problems.
\end{abstract}

Keywords Nonlinear thin film flow · Evolutionary cubic spline · Genetic algorithms · Sequential quadratic programming · Polynomial splines

Muhammad Asif Zahoor Raja

rajamaz@yuntec.edu.tw

Aamir Rizwan

17016109-001@uog.edu.pk

Iftikhar Ahmad

dr.iftikhar@uog.edu.pk

Muhammad Shoaib

dr.shoaib@cuiatk.edu.pk

1 Department of Mathematics, University of Gujrat, Gujrat, Pakistan

2 Future Technology Research Center, National Yunlin University of Science and Technology, 123 University Road, Section 3, Douliou, Yunlin 64002, Taiwan, R.O.C.

3 Department of Electrical and Computer Engineering, COMSATS University Islamabad, Attock Campus, Attock 43600, Pakistan

4 Department of Mathematics, COMSATS University Islamabad, Attock Campus, Attock 43600, Pakistan

\section{Introduction}

A well-known terminology "thin film" is commonly introduced for flow which is based on the layer of material and whose range lies from fractions of a nanometre to several micrometres in thickness. The flow spectrum is much smaller in one dimension than in the other (one or two dimensions). It is a characteristic that is used to reduce Navier-Stokes equations in a simple set of modelling equations. Flow-type problems with thin films (flow) are of main structural significance. Due to their numerous applications in different fields such as surface coating, chemical engineering, industrial processes, cooling, and the lubrication of heat exchanger fins into the motion of contact lens, the researchers are taking keen interest to work in this field [1].

In everyday life, problems of thin film flow range from very easy to complex affects, such as transferring a rain drop on an aircraft panel. It is admitted that in several procedures of industries, it also depends upon thin film flow. Thus, the 
research community is interested to work on various fields of natural science and engineering by using thin film flow. The most prominent applications related to industries are seen in the following fields such as paint coatings, modelling of dam breaking wave, and nuclear reactor drawn in fluid dynamics. Representation of dynamics of TFF is like tear films inside eye and in biophysics field like membranes. The characteristics of flow and thin film flow are most common. In addition, in the current advance and rapid age, thin film allows all those people to check the output of paints in surface flow covering, plan the design of nuclear reactor, and decrease the composition of machinery such as bearing fluid. Likewise, the biological applications of TFF are red blood cells flow across eyes, veins, and lungs friction [2].

The importance of flow that relates to the non-Newtonian fluids is much fruitful for physicians, applied mathematicians, and engineers. Furthermore, its involvement can be seen in different fields of engineering, social, and natural sciences. In many fields of nonlinear dynamic systems in technology and science, many researchers in these fluids consider the best analysis on the current situation. Examples include Sakiadis' [3] and Chen' [4] outstanding work exploring the movement of a viscous fluid across a moving solid surface or researching mixed convection flow across a porous medium [5]. Elahi and Riaz [6] studied the nonNewtonian MHD flow through viscous dissipation in a third grade fluid, examining the TFF of third grade fluid.

Many physical problems are nonlinear, but there is also inadequate linear analysis to properly explain the behaviour of physical systems. For nonlinear dynamic systems, an exact solution is always scarce, at least in the current state of science. New and creative methods capable of solving nonlinear system dynamics should be understood in this regard. Some valuable results for solving different nonlinear equations have recently been obtained. There are several analytical methods that refer to nonlinear problems, such as the weighted linearization process [7], Lindstedt-Poincare method [8], Adomian decomposition technique [9], boundary element technique [9], optimal perturbation homotopy method [10], optimal asymptotic homotopy method [11], etc. [12].

For weakly nonlinear problems, all of the methods listed above work very well and some of them work well, even for higher-order nonlinear systems. It is also very important to secure the convergence state of the solutions in the presence of highly nonlinear problems. For the nonlinear differential equation of thin film flow, we use the spline method in the current work to solve a boundary value problem. The fluid being used is third grade, which in the analysis of the problem introduces strong nonlinearities.

In order to achieve analytical approximations to the TFF field of a grade four fluid from outside of the large square cylinder, Siddiqui et al. [13] applied the homotopy analy- sis technique and the conventional perturbation approach. The steady flow of a fluid across porous plate was also addressed in [14]. Hayat and Sajid [15] established solution in the form of series for the same topic. The TFF of an inviscid third grade fluid across an inclined plane was studied by Mabood [16]. There are several other characteristics, such as time dependence, historical consequences, other nonlinear problems, and yield strain [17-19]. In the areas of industry and engineering, thin film flows have many implications. Thin film layers play an important role within chemical engineering in the growth of distillation columns, thin film reactions, evaporators, and condensers. A thin layer's enormous advantages depend on its minor density, which supplies its movement across microchannels. In physical engineering, thin film layers have essential roles and have wide applications in developing cooling techniques via heat sinks for nanotechnology. A lot of issues arise in geophysical engineering associated with thin film flows such as massive flooding, lava, and flash floods [20]. Thin film flow studies are related directly to Newtonian fluids in the available literature, and very little research has been reported on non-Newtonian fluids. These are also of great importance, as in the class of non-Newtonian fluids, shear thickening and pseudo-plastic or shear thinning liquids are perceived. The industrial sectors, photographic films, the deformation of polymer sheets, etc., studies of TFF of tensile liquids are of immense importance.

Non-Newtonian viscous fluid flow modelling equations arise to strongly nonlinear systems. The non-Newtonian liquids are widely used in trade and industry and now have become the subject of comprehensive research, particularly mixed with nanomaterials [21-28]. For instance, chemical industries handle polymers and plastics extensively, whereas rheological activity is used by biomedical devices such as homodialyser. For the mathematical model and TFF solution, a new nonlinear computing paradigm based on the CSA integrated with GAs aided by SQP, CSA-GA-SQP, is presented in this review. The main objective of such a model is to solve differential equations utilizing advanced stochastic solvers to optimize weights built on the hybrid technique of global research genetic algorithms with local search techniques. Many researchers use such techniques to solve linear and nonlinear differential equations [29-31,33-41,43,43]. Few recent applications include hybrid rotational nanofluidic model with thermal characteristic consideration [44], crosswise stream fluid model involving nanomaterial over porous stretching medium [45], mathematical models of hydrogen possessions [46], COVID-19 epidemical models with future generation disease control [47], and nonlinear corneal shape model [48].

Moreover, research community is interested in splines method to solve different types of differential equations in wide scale. Some examples of such approach are given in 
[49-55]. Recently, the spline method has been considered by the researchers as an ideal numerical method for solving many problems. For example, cubic B-spline approximation has been applied for approximate solution of the integrodifferential equation of fractional order [56], and solution of well-known stiff system arising in atomic physics is obtained using evolutionary optimized cubic splines [57]. TFF equation has been solved by using a new stochastically computational intelligent methodology constructed with the hybridization of active set and particle swarm optimization after transformation of mathematical models based on neural networks [58]. These facts discussed above provide the research community with the inspiration to explore, examine, and analyse stochastic paradigms to solve nonlinear singular TFF. For the TFF solution, a stochastic optimization computation paradigm focusing on CSA-GASQP was built in this research. The key aspects of current investigation may be expressed in terms of salient features as:

- A novel design of spline-evolutionary stochastic solver CSA-GA-SQP is presented for the analysis of TFF model of second grade fluid governed with second-order nonlinear differential system.

- The cubic spline approach (CSA) is exploited to transform the differential system for TFF model into an equivalent set of nonlinear equations.

- The approximation in mean squared error sense is incorporated for the formulation of figure of merit to solve the transform system of equations of TFF model.

- The optimization of the decision variables is conducted by global search efficacy of evolution by GAs integrated with sequential quadratic programming (SQP) for speedy adjustments.

- The designed spline-evolutionary computing paradigm, CSA-GA-SQP, is evaluated effectively for different scenarios of TFF model by variation of second grade and magnetic parameters, as well as variation in the length of splines.

The paper is organized in the following way: In Sect. 2, we construct the mathematical formulation of nonlinear thin film flow possible and apply the structured approach for evolutionary cubic spline interpolation with the appropriate overview of CSA-GAs and CSA-GA-SQP. In Sect. 3, we layout the design of the model and bring forth the numerical experiments of the CSA-GA-SQP through the study of various statistical analyses. Our results are presented in Sect. 5, whereas in the last section conclusion is presented.

\section{Mathematical Formulation}

The governing equations for incompressible fluid in the absence of thermal impacts are given in the following. For incompressible fluid, continuity equation takes the form

$\nabla \cdot V=0$.

The momentum equation is

$\rho \mathrm{D} V / \mathrm{D} t=-\nabla P+\rho f+\operatorname{div} \tau$,

where $\rho, V, P, \mathrm{D} / \mathrm{D} t$, and $\tau$ represent density, velocity, pressure, material derivative, and the stress tensor, respectively.

The third grade fluid is defined through the stress given as

$\tau=S_{1}+S_{2}+S_{3}$

$S_{1}=\mu B_{1}$

$S_{2}=\alpha_{1} B_{2}+\alpha_{2} B_{1}^{2}$

$S_{3}=\beta_{1} B_{3}+\beta_{2}\left(B_{1} B_{2}+B_{2} B_{1}\right)+\beta_{3}\left(\operatorname{tr} B_{2}\right) B_{1}$,

where $\alpha_{1}, \beta_{1}, \alpha_{2}, \beta_{2}, \beta_{3}$ are material constants and $\mu$ is the dynamic viscosity. Moreover, $B_{1}, B_{2}$, and $B_{3}$ are RivlinEricksen tensors.

$B_{n}=D B_{n-1} / D t+B_{n-1}(\nabla V)+(\nabla V)^{T} B_{n-1}, \quad n \geq 1$.

An incompressible thin film flow of third grade fluid along an inclined plane is considered. The fluid is flowing due to gravity as ambient air and surface tension are considered stationary along with uniform film thickness $\delta$. The velocity profile is given as

$V=(y(x), 0,0)$.

Substitutions of $V$ and $\tau$ in Eqs. 2-3 in the absence of pressure gradient to deal with TFF, and in general, nonlinear boundary value (BVP) thin film solution is obtained by the secondorder differential equation given as:[59]

$\frac{\mathrm{d}^{2} y}{\mathrm{~d} x^{2}}+\frac{6\left(\beta_{2}+\beta_{3}\right)}{\mu}\left(\frac{\mathrm{d} y}{\mathrm{~d} x}\right)^{2} \frac{\mathrm{d}^{2} y}{\mathrm{~d} x^{2}}-\frac{\rho g}{\mu}=0$,

having boundary conditions given as

$y=Y o, \quad x=0$,

$\frac{\mathrm{d} y}{\mathrm{~d} x}=0, \quad x=\delta$.

Here, $\mathrm{y}$ is the fluid velocity, bet $a_{2}$ and bet $_{3}$ are fluid content constants, $\mu$ represents the fluid density, $\rho$ is the mass, gravitational acceleration is represented by g, Yo is the velocity, and $\delta$ is the film's uniform thickness. 
We add a new parameter to create dimensionless as

$$
x^{*}=\frac{x}{\delta}, \quad y^{*}=\frac{y}{Y o}, \quad \beta=\frac{\left(\beta_{2}+\beta_{3}\right)}{\mu \delta^{2}}, \quad n=\frac{\rho g \delta^{2}}{\mu Y_{o}} .
$$

Then, it becomes(neglecting the symbol*)

$$
\frac{\mathrm{d}^{2} y}{\mathrm{~d} x^{2}}+6 \beta\left(\frac{\mathrm{d} y}{\mathrm{~d} x}\right)^{2} \frac{\mathrm{d}^{2} y}{\mathrm{~d} x^{2}}-n=0
$$

where $\beta$ is the material constant and $\mathrm{n}$ is the Stokes number. For Newtonian fluid $(\beta=0)$, exact solution of the above wellpositioned problem is given as

$y(x)=1-\frac{n}{2}\left(1-(1-x)^{2}\right)$.

The approximated solution for nonlinear system is expressed as:

$$
\begin{aligned}
y(x)= & 1-\frac{n}{2}\left(1-(1-x)^{2}\right)+\frac{n^{3} \beta}{2}\left(1-(1-x)^{4}\right) \\
& -2 n^{5} \beta^{2}\left(1-(1-x)^{6}\right)+12 n^{7} \beta^{3}\left(1-(1-x)^{8}\right) \\
& -88 n^{9} \beta^{4}\left(1-(1-x)^{1} 0\right)+362 n^{1} 1 \beta^{5}\left(1-(1-x)^{1} 2\right) .
\end{aligned}
$$

By using spline techniques, results are determined for Eq.

(4) and are compared with reference solution.

\section{Design Methodology}

Some important points of the proposed scheme are given that comprises two segments: Fitness function for TFFS is developed for optimal solution, while learning methodology structure is presented in the second part. The graphical abstract of the proposed scheme is shown in Fig. 1, and the flowchart of GA is presented in Fig. 2.

\subsection{Mathematical Modelling for Thin Film Flow Equation}

Necessary information in a compact form for the designed methodology is provided here to find the solution of the nonlinear TFF differential system. The goal of cubic spline interpolation is to get piecewise interpolation polynomials having continuous first and second derivatives, which consequently gives well-ordered interpolation-type function. The first derivative of cubic spline function is used for the slopes of the tangent lines which show the increasing or decreasing trend of the function, while curvature is determined by the second derivative of cubic spline function. Cubic splines are used for the mathematical system of TFF based on nonlinear differential equations to find the optimal solution with the following procedure; first of all, n-sub intervals [xi, xi+1] are obtained by dividing the domain of problem such as $i=1,2, \ldots, n$ and formulating a spline representation, for instance, as follows:

$f(x)=\left\{\begin{array}{c}f_{1}(x), x_{1} \leq x \leq x_{2} \\ f_{2}(x), x_{2} \leq x \leq x_{3} \\ f_{3}(x), x_{3} \leq x \leq x_{4} \\ \cdot \\ \cdot \\ \cdot \\ f_{n}(x), x_{n} \leq x \leq x_{n+1},\end{array}\right.$

where $\mathrm{f}(\mathrm{x})$ shows the optimal solution of the mathematical model for TFFS and $f_{i}(x)$ denotes $i$ th third-order polynomial spline as follows:

$$
f_{i}(x)=a_{i}+b_{i} x+c_{i} x^{2}+d_{i} x^{3}
$$

having the first derivative:

$f_{i}^{\prime}(x)=b_{i}+2 c_{i} x+3 d_{i} x^{2}$

and the second derivative:

$$
f_{i}^{\prime \prime}(x)=2 c_{i}+6 d_{i} x
$$

The objective is to find the optimal solution for TFFS by exploiting the efficacy of genetic algorithms (GAs) and sequential quadratic programming soft computing infrastructures. The universal estimated proficiency of GAs is used to develop the unsupervised mathematical framework for TFFS. GAs is efficient and reliable approach for finding the accurate solution searching through the global minima of the proposed function which can also avoid the divergence. And optimization performance is further improved by the process of hybridization with sequential quadratic programming (SQP). Thus, in the present study optimization of the objective function model with CSA is carried out initially with the GAs procedure which satisfies the boundary conditions associated with the mathematical model as a global search algorithm aided with local search technique of SQP, i.e. CSA-GA-SQP.

Consider the differential equation

$£\left[x, y, y^{\prime}, y^{\prime \prime}\right]=0$.

Now, the splines model the differential system in the input interval, so for $\mathrm{i}=1,2, \ldots, \mathrm{p}$ :

$£\left[x, f_{i}(x), f_{i}^{\prime}(x), f_{i}^{\prime \prime}(x)\right]=0 ; \quad x \in\left[x_{i}, x_{i+1}\right]$. 




Fig. 1 Graphical illustration of the CSA-GA-SQP for nonlinear singular TFF

Also, the first and last spline function should satisfy the end The $\mathrm{f}(\mathrm{x})$ must be continuous: boundary conditions

$f_{1}\left(x_{1}\right)=y_{1}$

and

$f_{n-1}\left(x_{n}\right)=y_{n}$.

$$
f_{i}(x)=f_{i-1}(x) ; \quad i=2,3,4, \ldots \ldots, p .
$$

The first derivative of $f(x)$ should be continuous:

$f_{i}^{\prime}(x)=f_{i-1}^{\prime}(x) ; \quad i=2,3,4, \ldots \ldots ., p$. 



Fig. 2 Flowchart for CSA-GA-SQP

The continuity of curvature implies:

$f_{i}^{\prime \prime}(x)=f_{i-1}^{\prime \prime}(x) ; \quad i=2,3,4, \ldots \ldots ., p$.

\section{Application to Thin Film Flow Equation}

(21) The general form of TFF is given as

$$
\frac{\mathrm{d}^{2} y}{\mathrm{~d} x^{2}}+6 \beta\left(\frac{\mathrm{d} y}{\mathrm{~d} x}\right)^{2} \frac{\mathrm{d}^{2} y}{\mathrm{~d} x^{2}}-n=0
$$


with boundary conditions

$$
\begin{aligned}
y & =1, \quad x=0, \\
\frac{\mathrm{d} y}{\mathrm{~d} x} & =0, \quad x=1 .
\end{aligned}
$$

The splines are incorporated along with first and second derivatives for the solution of TFFS, where the optimal solution is shown by $\mathrm{f}$.

$$
\begin{aligned}
y & =f_{i}(x) \\
& =\sum_{p=0}^{3} b_{i, p} x^{p} ; x \in\left[x_{i}, x_{i+1}\right], i=[1, m-1], \\
y^{\prime} & =f_{i}^{\prime}(x) \\
& =\sum_{p=0}^{3} p b_{i, p} x^{p-1} ; x \epsilon\left[x_{i}, x_{i+1}\right], i=[1, m-1], \\
y^{\prime \prime} & =f_{i}^{\prime \prime}(x) \\
& =\sum_{p=0}^{3} p(p-1) b_{i, p} x^{p-2} ; x \epsilon\left[x_{i}, x_{i+1}\right], i=[1, m-1],
\end{aligned}
$$

where $f_{i}$ represents $i$ th spline solution and $b_{i, p}$ represents $p$ th coefficients of $i$ th spline.

\subsection{Soft Computing}

Soft computing infrastructure is of great significance for artificial intelligence algorithm and procedures of learning. A variety of optimization procedures based on bio- and natureinspired computing algorithms, fuzzy logic, and supervised and unsupervised artificial neural networks are supported by soft computing system. Physical and mathematical models comprising linear and nonlinear stiff differential equations can be handled by soft computing infrastructures with certain tolerance for incorrectness and unpredictability to find the low-cost optimal results. Soft computing infrastructure is also used to handle the fitness functions for different natureand bio-inspired techniques in the process of optimization. Zadeh [60] is the pioneer in the field of soft computing; through this new development, the tolerances of the objective functions for different evolutionary- and nature-inspired optimization techniques are investigated for incorrectness and unpredictability to achieve low-cost functions and trace abilities of the optimal solutions. Soft computing infrastructures are helpful to exploit the error functions in terms of GAs which were introduced by John Henry Holland in seventies of nineteenth century $[61,62]$, that is based on evolution, which is the mechanism of survival of the best. GAs working is based on four important components, i.e. selection of initial population, reproduction in the considered population, crossover, and mutation. GAs are based on initial population
Table 1 Settings for GAs optimization tools

\begin{tabular}{ll}
\hline Index setting & \\
\hline Solver & GA \\
Individuals in population & 200 \\
Total number of generations & 200 \\
Crossover scheme & Heuristic routine \\
Fraction of crossover & 0.2 \\
Function tolerance & $1.00 \mathrm{E}-20$ \\
Range initialization & {$[-1 ; 1]$} \\
Selection scheme & stochastic uniform \\
Scaling procedure & Ranking \\
Elitism & 20 counts \\
Mutation scheme & Adaptive feasible \\
Other & Defaults \\
\hline
\end{tabular}

Table 2 Parameter setting for SQP

\begin{tabular}{ll}
\hline Index setting & \\
\hline Solver & 'FMINCON' \\
Method & SQP \\
Initial weight & Best of GAs \\
Iterations & 2000 \\
X-tolerance & $1.00 \mathrm{E}-12$ \\
Maximum function counts & $2.00 \mathrm{E}+05$ \\
Function tolerance & $1.00 \mathrm{E}-06$ \\
Constraints tolerance & 0 \\
Nonlinear constraints & $1.00 \mathrm{E}-11$ \\
Hessian & Off \\
SQP constraints & Off \\
\hline
\end{tabular}

for the optimization procedure to find the best combinations from the given initial population for the best optimal solution. The initial population considered for the solution of the model is candidate solution. Selection of initial population is of great importance as for small initial population it can be the premature convergence, while against a large size of population the expensive computation procedure is involved. Therefore, the following steps can be implemented for finding the optimal solution.

\section{(a) Initialization.}

Candidate solutions are initiated randomly keeping in view the model-related information.

\section{(b) Fitness Evaluations}

The fitness function is developed in such a way that it provides the solution close to optimal results for the proposed system model. Fitness for all candidate solutions is checked and endorsed by the designed fitness function. 



Fig. 3 Learning curves of CSA-GA for the proposed problems with 5 splines


Score Histogram


Fig. 4 Learning curves of CSA-GA for the proposed problems with 10 splines

\section{(c) Selection}

More better and close values to the actual solutions based on higher fitness are allocated along with the survival of the fittest mechanism. In this section, potential solutions with close resemblance to the optimal values are preferred than worse solutions. The chromosomes with high-rank of fitness in the existing population will survive as a parent for the next generation.

(d) Recombination

Solutions with better fitness are produced by the combination of pairwise adjusted potential solutions. Mechanism on the basis of recombination setting is established for the 



Fig. 5 Learning curves of CSA-GA for the proposed problems with 15 splines


Fig. 6 Learning curves of CSA-GA-SQP for the proposed problems with 5 splines

optimal solutions related to the proposed problem. Offspring under the process of recombination should not be similar to any parent, but traits are transferred in a unique way as per procedure designed by Goldberg.

\section{(e) Mutations}

New genes are replaced which were unremembered under the procedure of recombination and production new offspring and then randomly modified by recombination operator on parental chromosomes. There are different variants of mutation operator, but it mostly influences the individual's traits 



Step Size: $1.82617 \mathrm{e}-10$

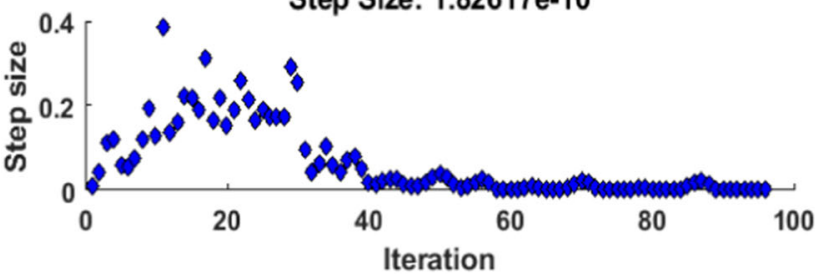

Total Function Evaluations: 7918
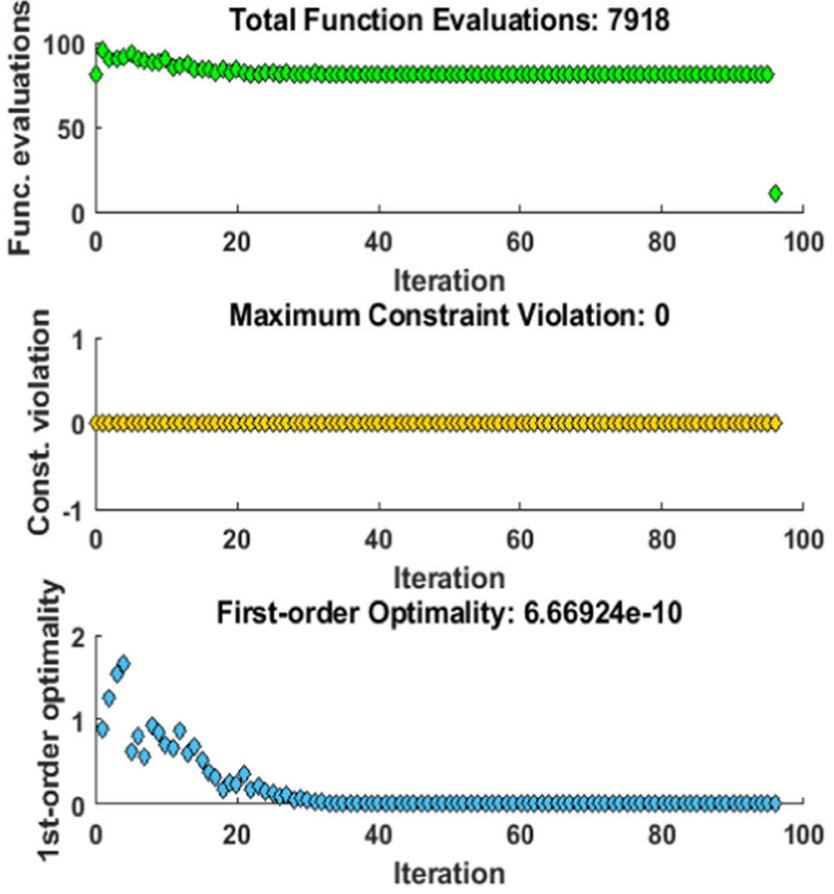

Fig. 7 Learning curves of CSA-GA-SQP for the proposed problems with 10 splines
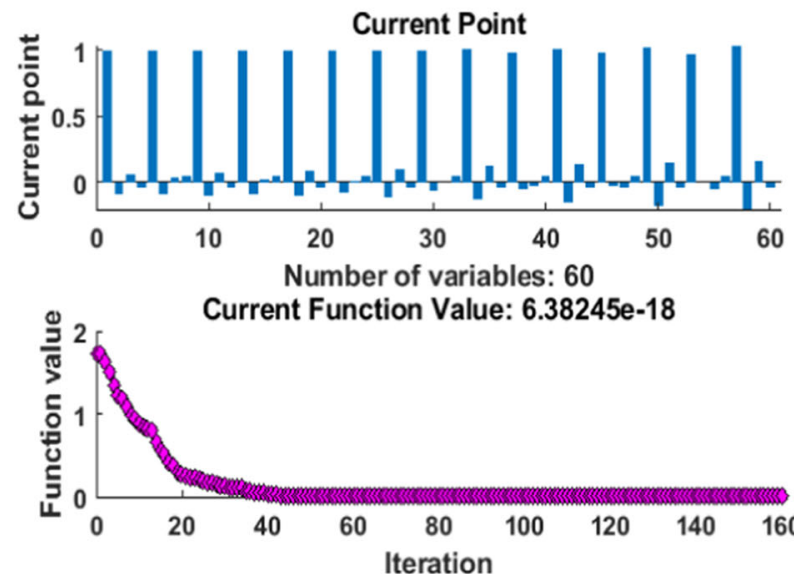

Step Size: $2.18454 \mathrm{e}-10$





Maximum Constraint Violation: 0


Fig. 8 Learning curves of CSA-GA-SQP for the proposed problems with 15 splines

with new alterations for optimal solutions. Mutation influences the candidate solution in the vicinity possessing the most favourable and reliable potential candidate solutions.

\section{(f) Replacements}

Variants of replacement operator based on generationwise replacement, elitist replacement, and time-independent replacements are employed in genetic algorithms. Original parental population is replaced by creating offspring through selection, recombination, and mutation procedures. Note: Repeat steps 2-6 until a terminating condition is met.

(g) Termination Criteria

The GAs terminates if the following common termination conditions are satisfied: 

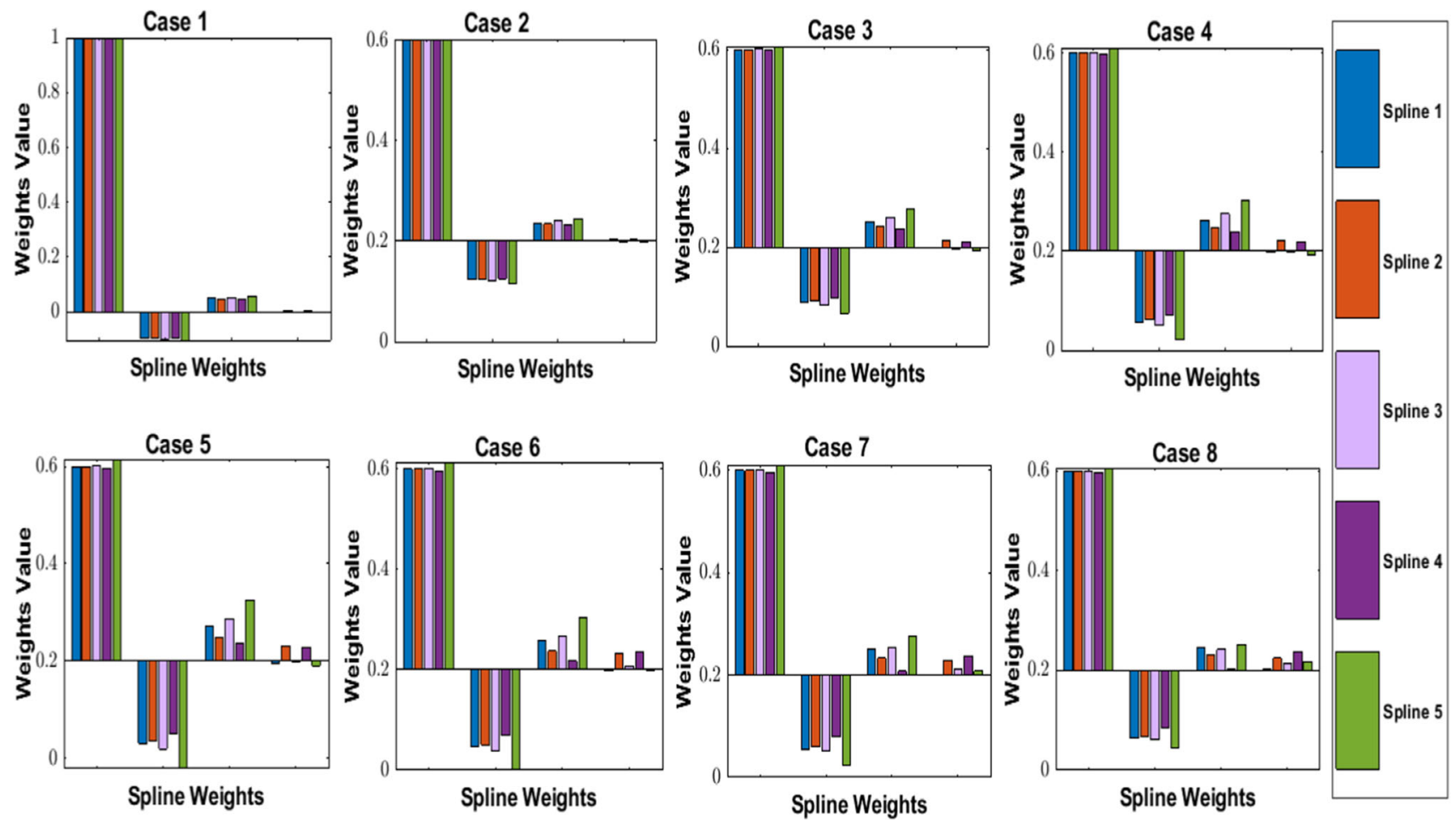

Fig. 9 The best weights of 5 splines for cases $1-8$

\section{(h) Local Search Fine Tuning}

After selecting the appropriate individual by GAs, it fed into the SQP algorithms as a beginning stage for adjusting and improvement.

The optimization arrangements for GA and GA-SQP are presented in Tables 1 and 2.

\section{Numerical Experimentation with Discussion}

The detailed numerical simulations with results for presented CSA-GA-SQP solver are provided here for the analysis of nonlinear thin film flow model. The three scenarios on the basis of length of splices for CSA, i.e. 5, 10, and 15 splinesbased model, are executed in interval $(0,1)$.

\subsection{Scenario 1: 5 Splines-Based CSA-GA-SQP for Thin Film Flow Model}

The thin film flow equation for the case based on input intervals along with boundary conditions is written as:

$$
\begin{aligned}
& \frac{\mathrm{d}^{2} y}{\mathrm{~d} x^{2}}+6 \beta\left(\frac{\mathrm{d} y}{\mathrm{~d} x}\right)^{2} \frac{\mathrm{d}^{2} y}{\mathrm{~d} x^{2}}-n=0 \quad y(0)=1, \\
& y^{\prime}(1)=0 \quad x \in[0,1] .
\end{aligned}
$$

The figure of merit or objective function for the said case is written as follows:

$\epsilon=\sum_{k=0}^{5} \epsilon_{k}$

where

$$
\begin{aligned}
& \epsilon_{o}=\sum_{i=1}^{5} f_{i}^{\prime \prime}+6 \beta\left(f_{i}^{\prime}\right)^{2} f_{i}^{\prime \prime}-n, \\
& \epsilon_{1}=\sum_{i=1}^{5}\left(f_{i}-f_{i-1}\right)^{2}, \\
& \epsilon_{2}=\sum_{i=1}^{5}\left(f_{i}^{\prime}-f_{i-1}^{\prime}\right)^{2}, \\
& \epsilon_{3}=\sum_{i=1}^{5}\left(f_{i}^{\prime \prime}-f_{i-1}^{\prime \prime}\right)^{2}, \\
& \epsilon_{4}=\sum_{i=1}^{5}\left(f_{1}(1)-0\right)^{2}, \\
& \epsilon_{5}=\sum_{i=1}^{5}\left(f_{1}^{\prime}(0)-1\right)^{2} .
\end{aligned}
$$

Accordingly, the objective/cost function for 10 and 15 splines is constructed. The RE for $\mathrm{p}=5$ splines is mathematically 


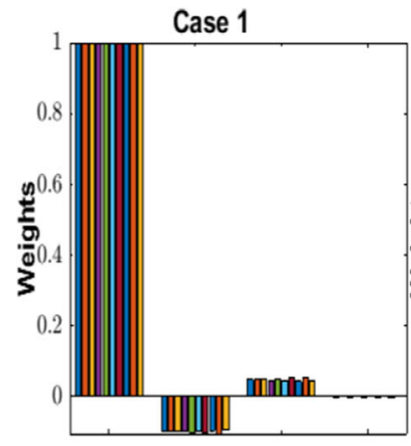

Spline Weights

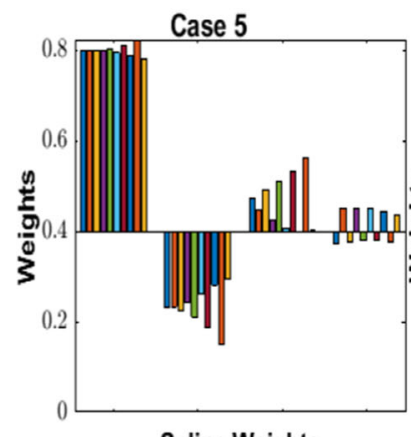

Spline Weights

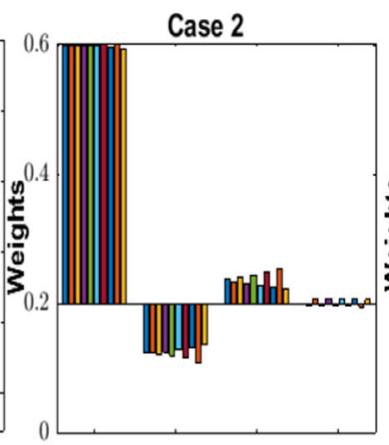

Spline Weights

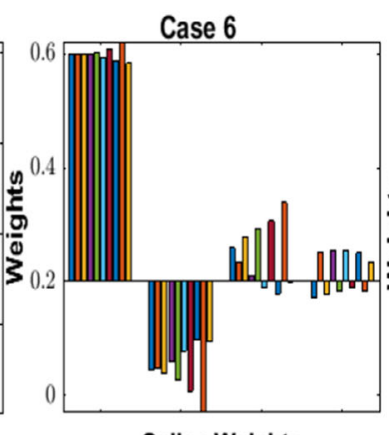

Spline Weights

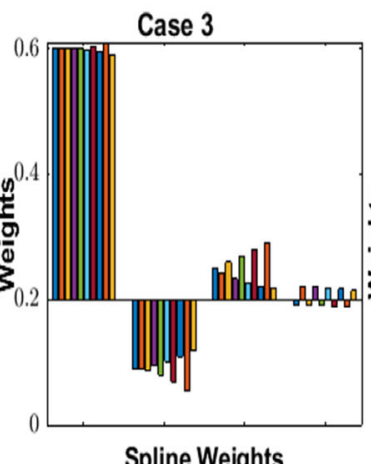

Spline Weights

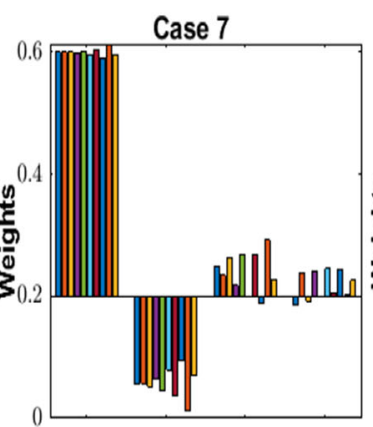

Spline Weights

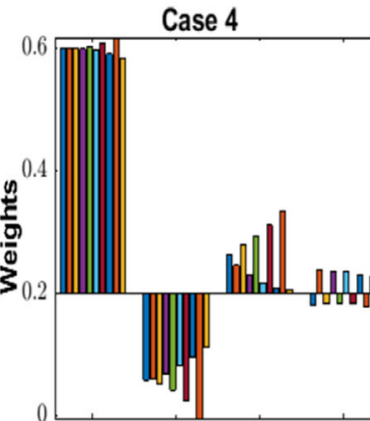

Spline Weights

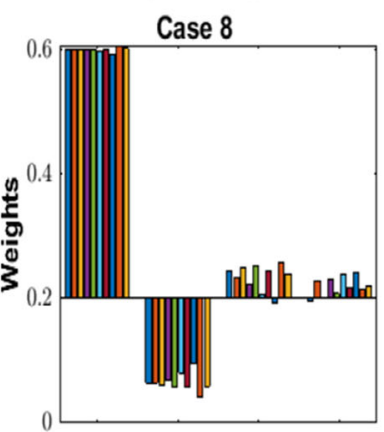

Spline Weights

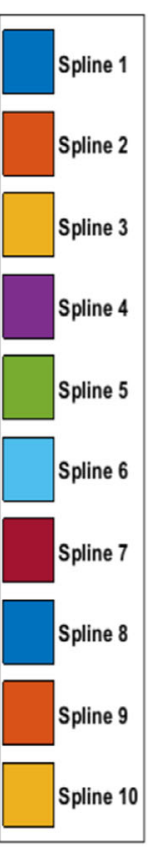

Fig. 10 The best weights of 10 splines for cases $1-8$
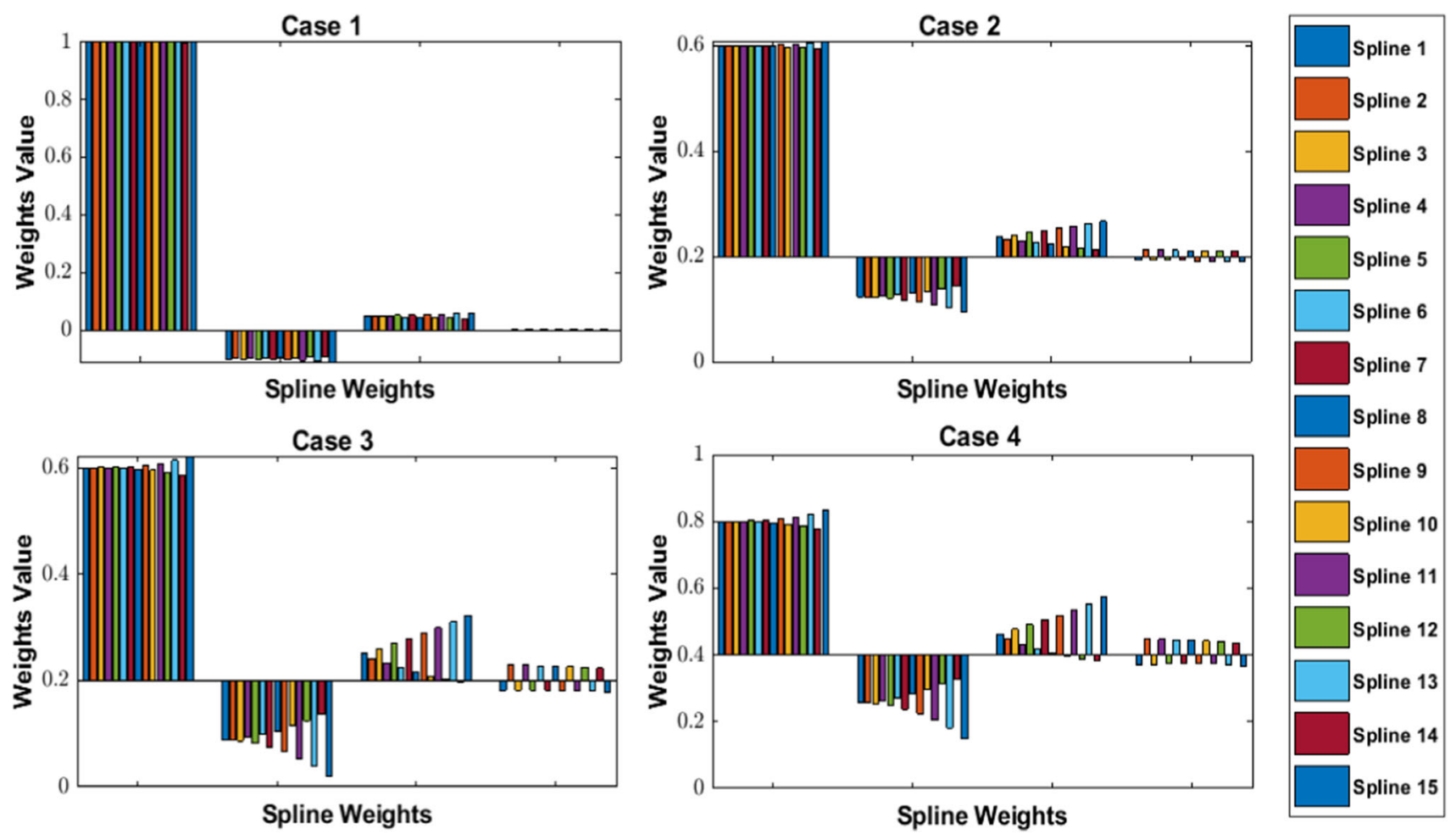

Fig. 11 The best weights of 15 splines for cases 1-4 

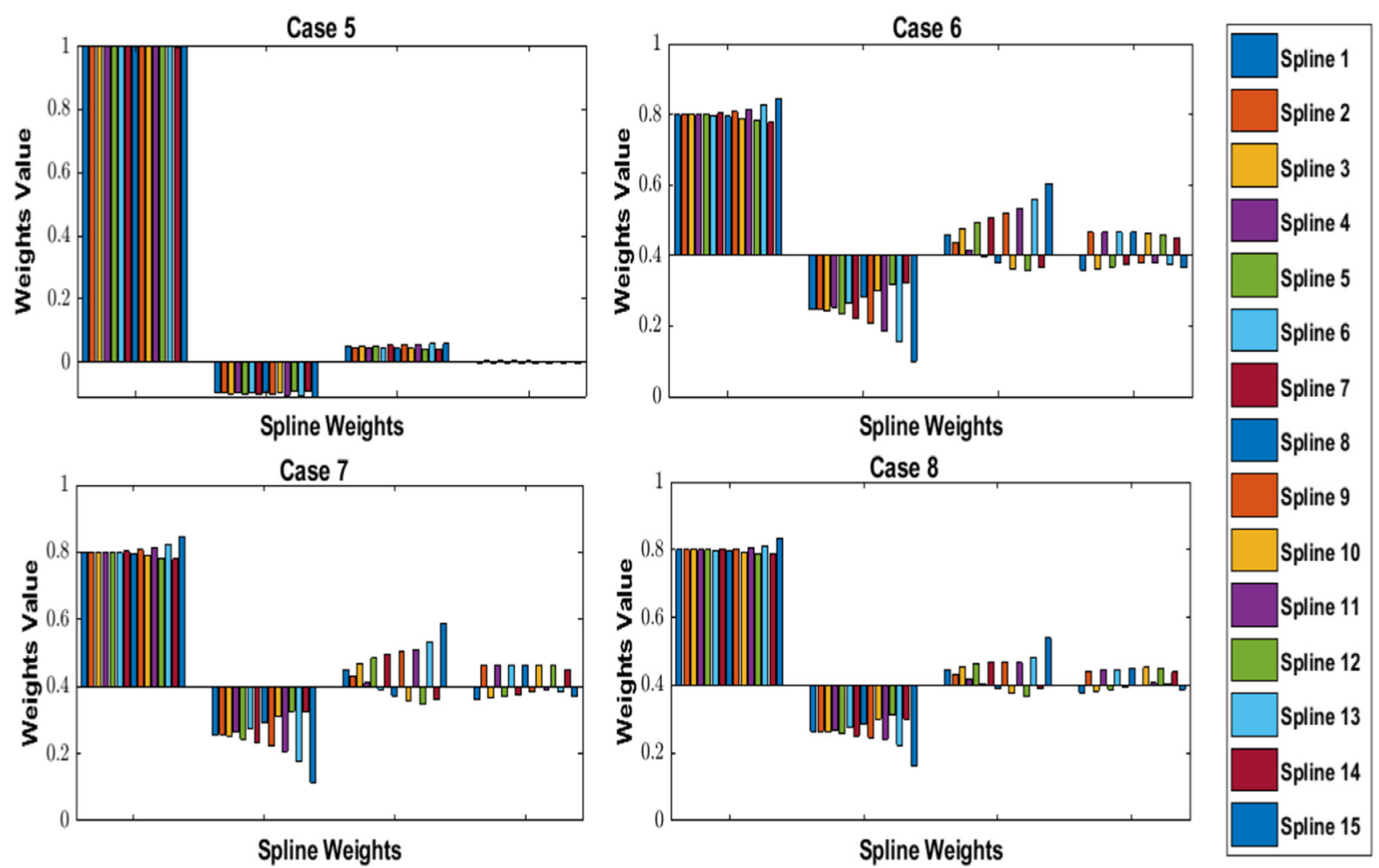

Fig. 12 The best weights of 15 splines for cases 5-8

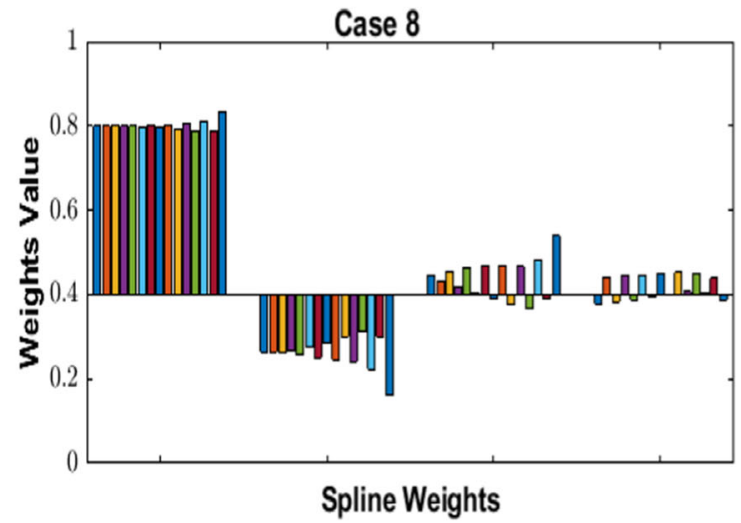

Spline 9

Spline 10

Spline 11

Spline 12

Spline 13

Spline 14

Spline 15

represented as follows:

$R E=\sum_{i=1}^{5}\left|y_{i, j}^{\prime \prime}+6 \beta\left(y_{i, j}^{\prime}\right)^{2} y_{i, j}^{\prime \prime}-n\right|$.

If $x \in[0,1]$, then $h=0.1$, where $y_{i, j}$ represent the solution of $i$ th sub-spline for $j$ th input in accordance with step size h.

\subsection{Scenario 2: 10 Splines-Based CSA-GA-SQP for Thin Film Flow Model}

The fitness function is formulated same as scenario 1 for 10 splines by taking $i=1$ to $i=10$ and for RE $p=10$.

\subsection{Scenario 3: 15 Splines-Based CSA-GA-SQP for Thin Film Flow Model}

The fitness function is formulated same as scenario 1 for 15 splines by taking $i=1$ to $i=15$ and for RE $p=15$.

\subsection{Performance Analysis CSA-GA-SQP for Multi-Runs}

All three scenarios are optimized through memetic computing based on GA-SQP algorithm as presented in Sect. 3. The learning curves for 5,10 , and 15 splines in case of GA and GA-SQP are presented in Figs. 3, 4, 5, 6, 7, and 8, and their best weights are shown in Figs. 9, 10, 11, and 12 .

- Solution is satisfied minimum criteria.

- Change in the form of no improvement in the population after some iterations.

- Attain the specific criteria regarding generations.

- Goal defined for objective function is achieved.

Process of simulations has been completed for the solution of thin film flow problems through the proposed scheme. The numerical results for these problems are determined through Mathematica and compared with proposed numerical results in MATLAB. The complete statistical analysis of all cases for different splines 5, 10, and 15 is given in Tables 3, 4, 5, 6, 7 , and 8 . Further, graphical illustration of numerical solutions for different splines 5, 10, and 15 is shown in Figs. 13, 14, and 15. Table 9 shows the variation of nondimensional material constant and Stokes number. 


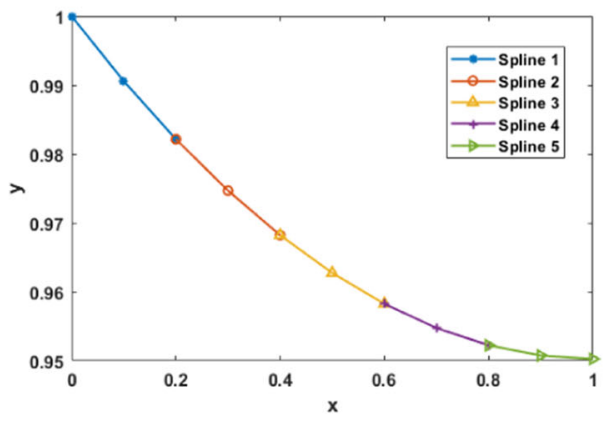

(a) Case 1 for $\beta=0.5, \mathrm{n}=0.1$

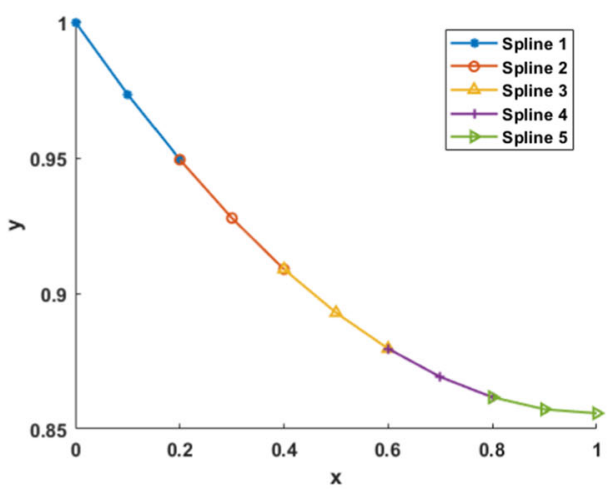

(c) Case 3 for $\beta=0.5, \mathrm{n}=0.3$

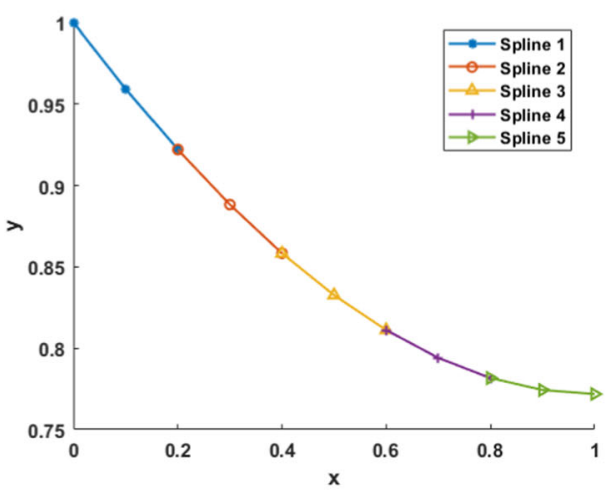

(e) Case 5 for $\beta=0.5, \mathrm{n}=0.5$

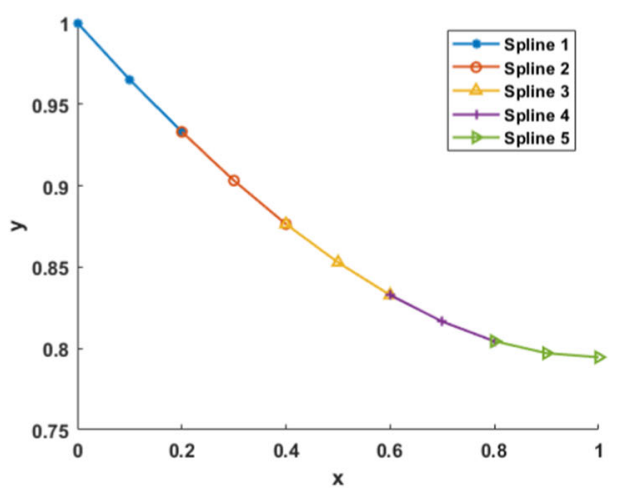

(g) Case 7 for $\beta=1.5, \mathrm{n}=0.5$

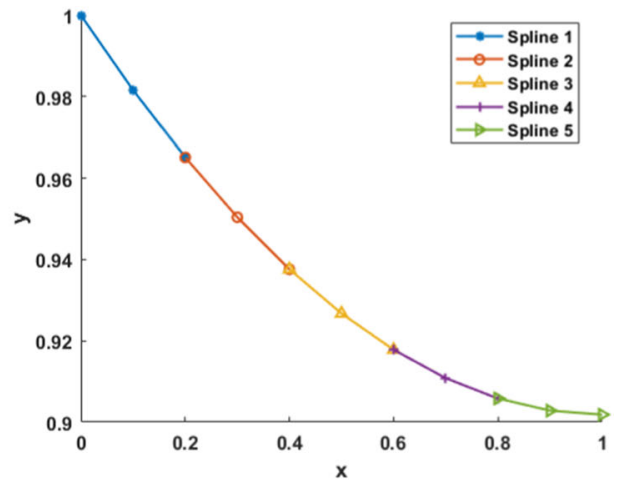

(b) Case 2 for $\beta=0.5, \mathrm{n}=0.2$

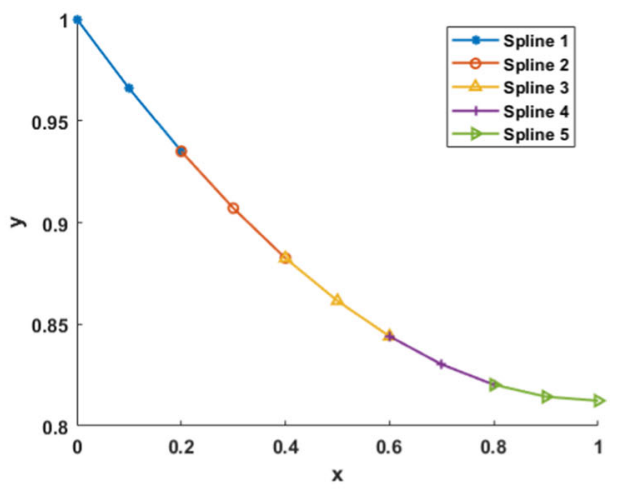

(d) Case 4 for $\beta=0.5, \mathrm{n}=0.4$

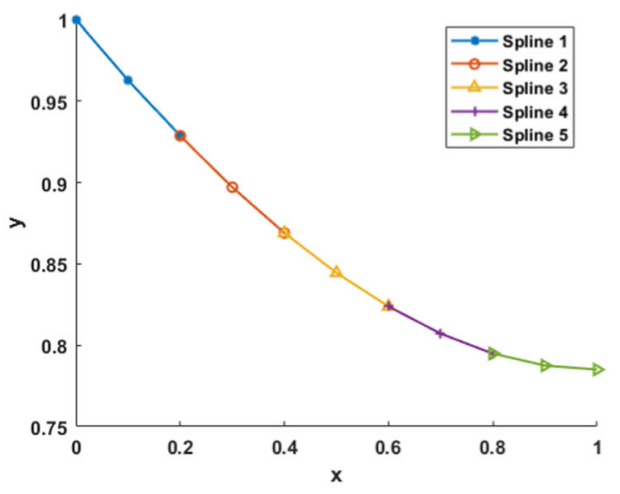

(f) Case 6 for $\beta=1.0, \mathrm{n}=0.5$

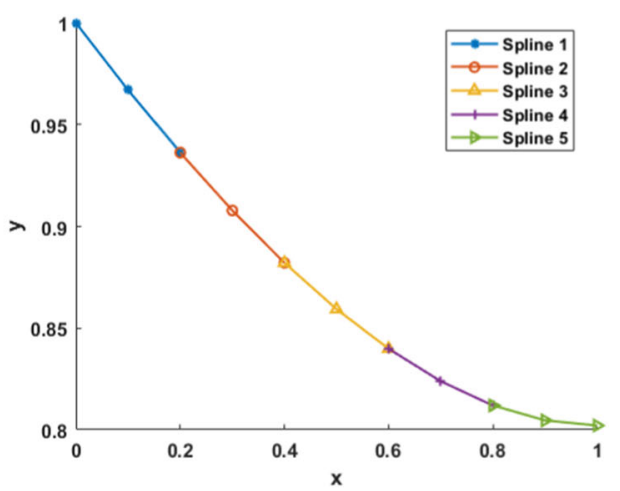

(h) Case 8 for $\beta=2.0, \mathrm{n}=0.5$

Fig. 13 Spline fitting of CSM-GA-SQP for different cases of TFF problem with 5 splines 


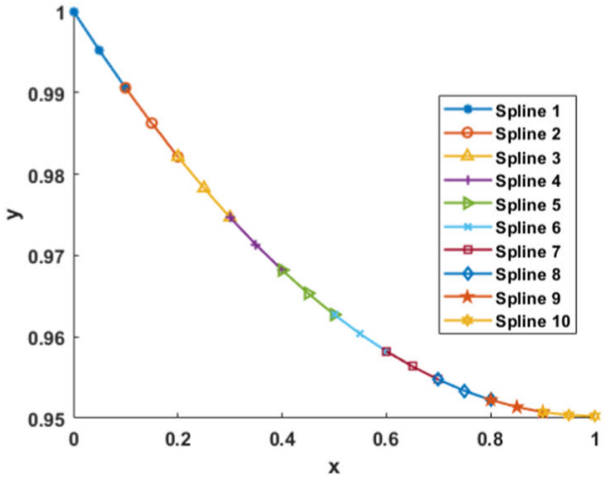

(a) Case 1 for $\beta=0.5, \mathrm{n}=0.1$

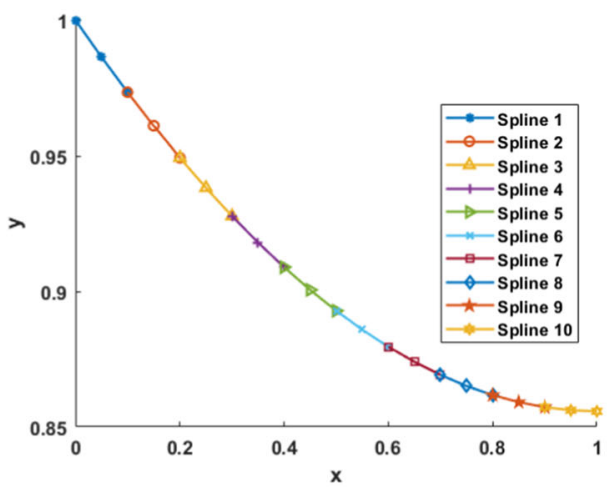

(c) Case 3 for $\beta=0.5, \mathrm{n}=0.3$

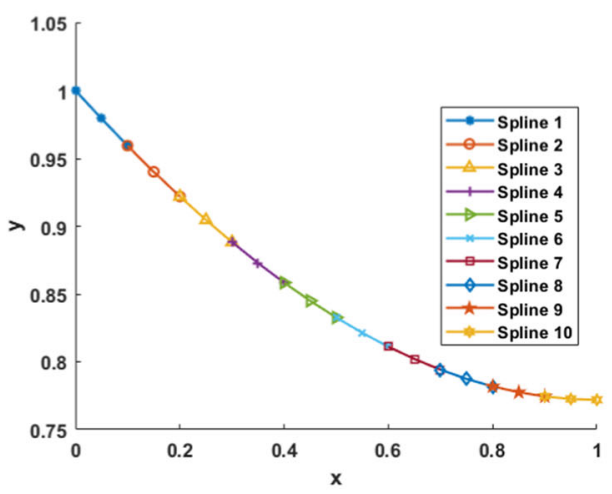

(e) Case 5 for $\beta=0.5, \mathrm{n}=0.5$

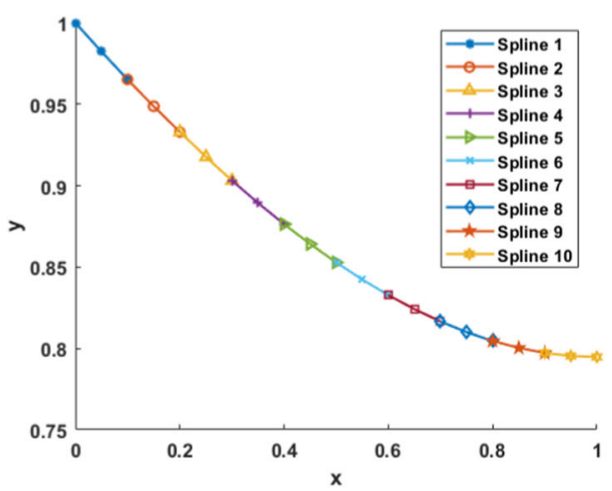

(g) Case 7 for $\beta=1.5, \mathrm{n}=0.5$

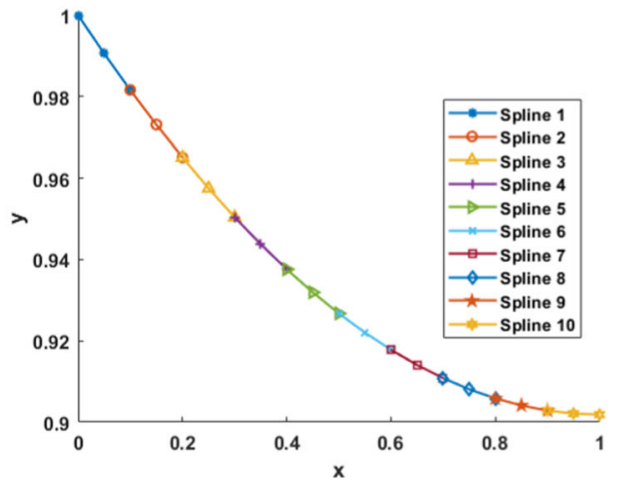

(b) Case 2 for $\beta=0.5, \mathrm{n}=0.2$

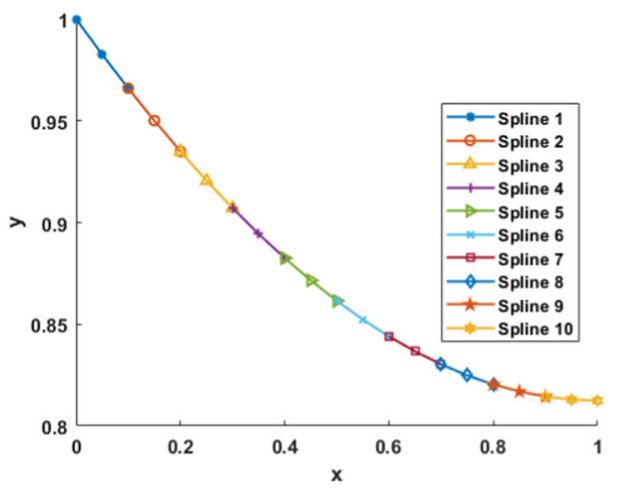

(d) Case 4 for $\beta=0.5, \mathrm{n}=0.4$

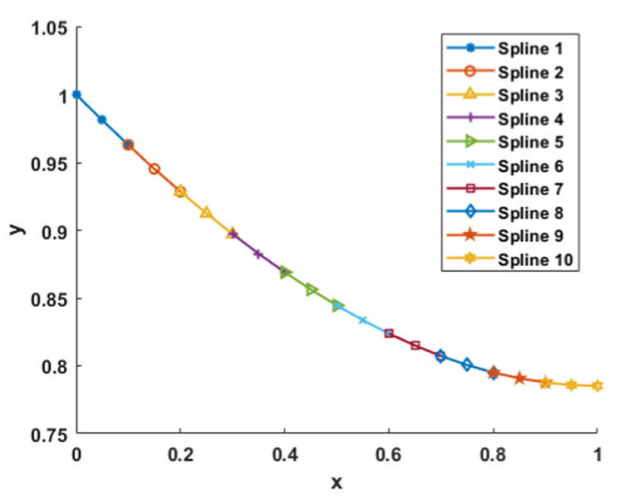

(f) Case 6 for $\beta=1.0, \mathrm{n}=0.5$

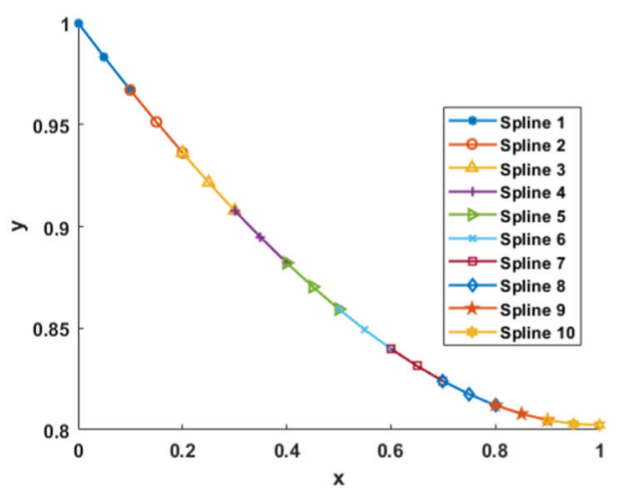

(h) Case 8 for $\beta=2.0, \mathrm{n}=0.5$

Fig. 14 Spline fitting of CSM-GA-SQP for different cases of TFF problem with 10 splines 


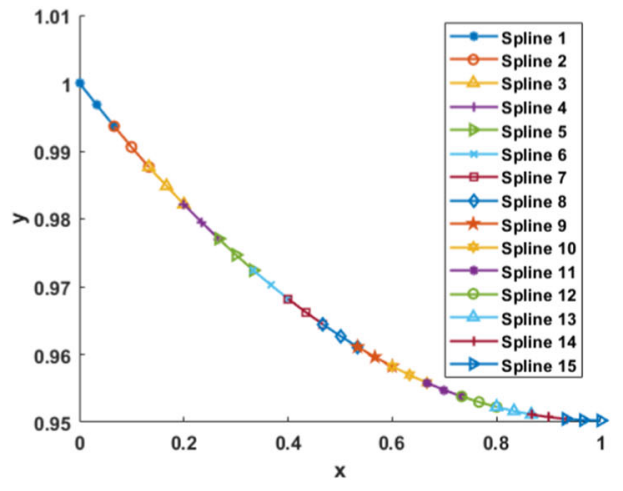

(a) Case 1 for $\beta=0.5, \mathrm{n}=0.1$

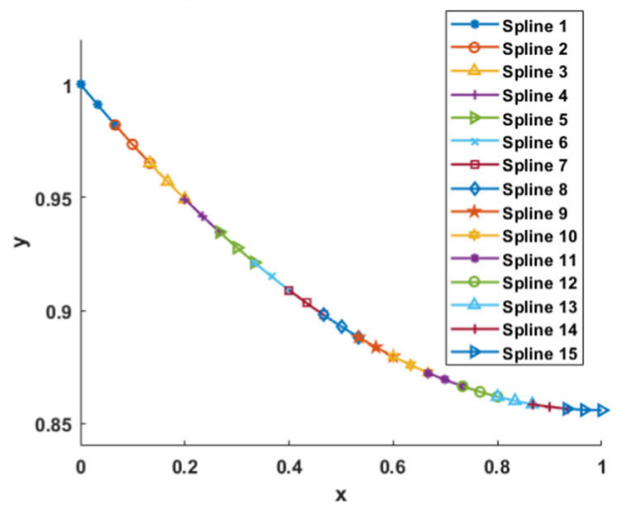

(c) Case 3 for $\beta=0.5, \mathrm{n}=0.3$

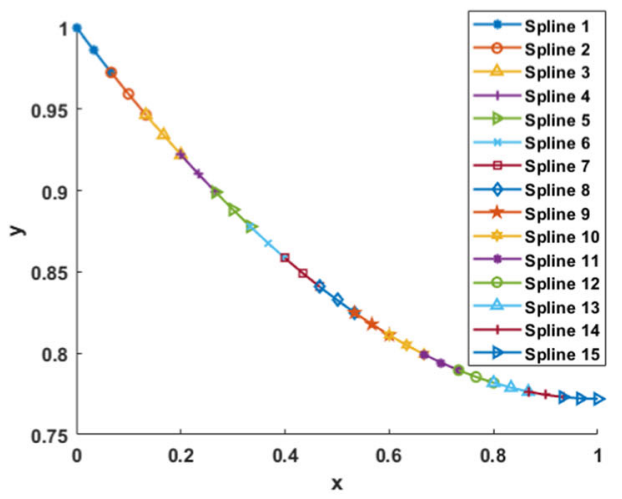

(e) Case 5 for $\beta=0.5, \mathrm{n}=0.5$

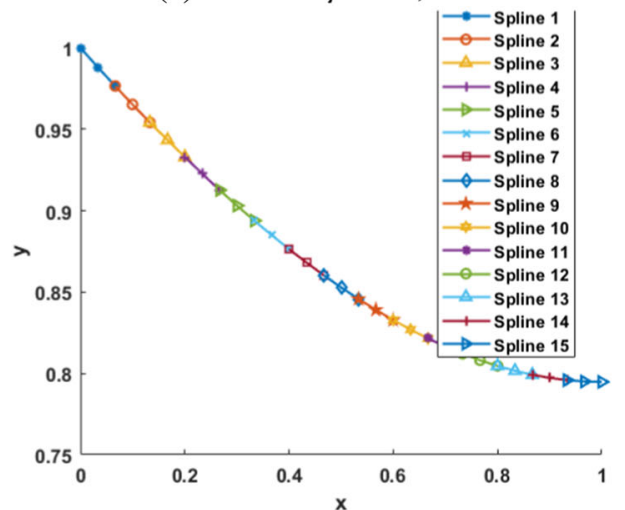

(g) Case 7 for $\beta=1.5, \mathrm{n}=0.5$

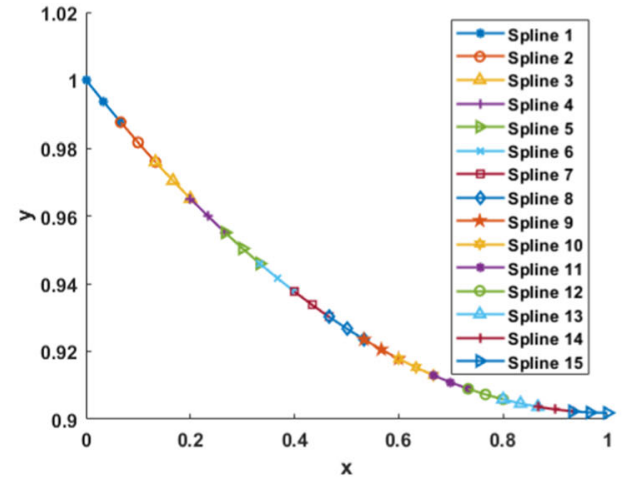

(b) Case 2 for $\beta=0.5, \mathrm{n}=0.2$

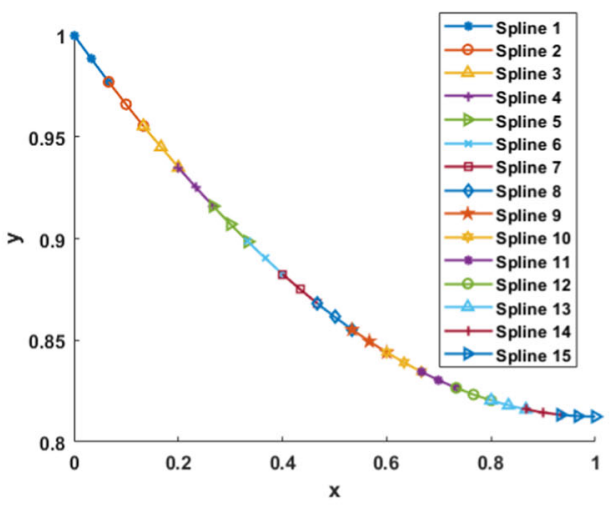

(d) Case 4 for $\beta=0.5, \mathrm{n}=0.4$

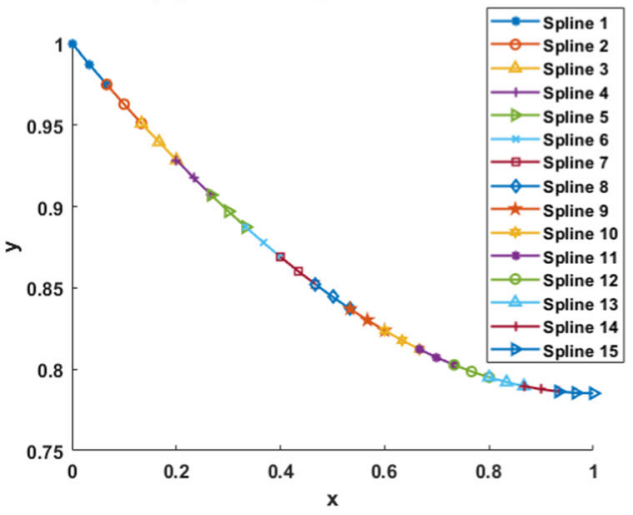

(f) Case 6 for $\beta=1.0, \mathrm{n}=0.5$

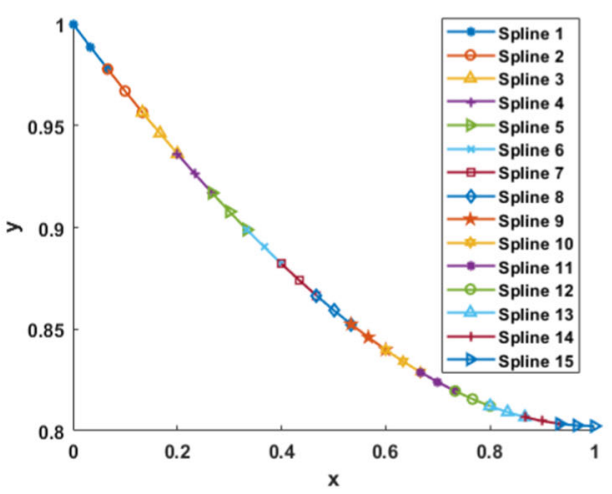

(h) Case 8 for $\beta=2.0, \mathrm{n}=0.5$

Fig. 15 Spline fitting of CSM-GA-SQP for different cases of TFF problem with 15 splines 
Table 3 Relative study on the basis of absolute errors of GA for spline 5

\begin{tabular}{|c|c|c|c|c|}
\hline Case & Max & Min & Mean & Std \\
\hline \multirow[t]{5}{*}{1} & $6.97 * 10-06$ & $7.11 * 10-13$ & $3.37 * 10-06$ & $3.49 * 10-06$ \\
\hline & $6.97 * 10-06$ & $1.70 * 10-06$ & $3.87 * 10-06$ & $2.75 * 10-06$ \\
\hline & $4.38 * 10-06$ & $4.09 * 10-07$ & $2.58 * 10-06$ & $2.01 * 10-06$ \\
\hline & $4.73 * 10-06$ & $1.75 * 10-07$ & $3.09 * 10-06$ & $2.53 * 10-06$ \\
\hline & $4.73 * 10-06$ & $2.40 * 10-07$ & $2.97 * 10-06$ & $2.40 * 10-06$ \\
\hline \multirow[t]{5}{*}{2} & $1.32 * 10-05$ & $1.06 * 10-12$ & $6.69 * 10-06$ & $6.61 * 10-06$ \\
\hline & $1.90 * 10-05$ & $2.14 * 10-06$ & $1.15 * 10-05$ & $8.58 * 10-06$ \\
\hline & $1.90 * 10-05$ & $2.66 * 10-06$ & $1.09 * 10-05$ & $8.19 * 10-06$ \\
\hline & $3.08 * 10-05$ & $2.66 * 10-06$ & $1.68 * 10-05$ & $1.41 * 10-05$ \\
\hline & $3.08 * 10-05$ & $9.48 * 10-06$ & $2.03 * 10-05$ & $1.07 * 10-05$ \\
\hline \multirow[t]{5}{*}{3} & $5.52 * 10-05$ & $4.77 * 10-12$ & $2.73 * 10-05$ & $2.76 * 10-05$ \\
\hline & $5.52 * 10-05$ & $5.86 * 10-06$ & $3.54 * 10-05$ & $2.61 * 10-05$ \\
\hline & $4.51 * 10-05$ & $7.96 * 10-06$ & $2.42 * 10-05$ & $1.90 * 10-05$ \\
\hline & $7.96 * 10-05$ & $7.96 * 10-06$ & $4.11 * 10-05$ & $3.61 * 10-05$ \\
\hline & $7.96 * 10-05$ & $1.34 * 10-05$ & $4.67 * 10-05$ & $3.31 * 10-05$ \\
\hline \multirow[t]{5}{*}{4} & $8.90 * 10-05$ & $7.19 * 10-12$ & $4.39 * 10-05$ & $4.45 * 10-05$ \\
\hline & $8.90 * 10-05$ & $1.09 * 10-05$ & $5.70 * 10-05$ & $4.09 * 10-05$ \\
\hline & $7.10 * 10-05$ & $5.02 * 10-07$ & $3.61 * 10-05$ & $3.52 * 10-05$ \\
\hline & 0.000142 & $5.02 * 10-07$ & $7.15 * 10-05$ & $7.10 * 10-05$ \\
\hline & 0.000142 & $4.96 * 10-05$ & $9.66 * 10-05$ & $4.64 * 10-05$ \\
\hline \multirow[t]{5}{*}{5} & 0.000137 & $1.59 * 10-11$ & $6.78 * 10-05$ & $6.87 * 10-05$ \\
\hline & 0.000137 & $2.97 * 10-05$ & $8.49 * 10-05$ & $5.39 * 10-05$ \\
\hline & $8.78 * 10-05$ & $5.29 * 10-06$ & $4.54 * 10-05$ & $4.13 * 10-05$ \\
\hline & 0.000207 & $5.29 * 10-06$ & 0.000104 & 0.000101 \\
\hline & 0.000207 & $8.76 * 10-05$ & 0.000149 & $5.98 * 10-05$ \\
\hline \multirow[t]{5}{*}{6} & 0.00014 & $1.13 * 10-11$ & $6.90 * 10-05$ & $6.98 * 10-05$ \\
\hline & 0.00014 & $4.55 * 10-05$ & $8.19 * 10-05$ & $5.06 * 10-05$ \\
\hline & $6.06 * 10-05$ & $1.75 \mathrm{~V} * 10-06$ & $3.12 * 10-05$ & $2.94 * 10-05$ \\
\hline & 0.000224 & $1.75 * 10-06$ & 0.000111 & 0.000111 \\
\hline & 0.000224 & 0.000162 & 0.000195 & $3.12 * 10-05$ \\
\hline \multirow[t]{5}{*}{7} & 0.00012 & $3.54 * 10-11$ & $5.92 * 10-05$ & $6.00 * 10-05$ \\
\hline & 0.00012 & $3.24 * 10-05$ & $6.76 * 10-05$ & $4.62 * 10-05$ \\
\hline & $3.24 * 10-05$ & $2.26 * 10-06$ & $1.66 \mathrm{~V} * 10-05$ & $1.51 * 10-05$ \\
\hline & 0.00021 & $2.26 * 10-06$ & 0.000103 & 0.000104 \\
\hline & 0.000219 & 0.00021 & 0.000214 & $4.61 \mathrm{~V} * 10-0$ \\
\hline \multirow[t]{5}{*}{8} & $9.55 * 10-05$ & $3.78 * 10-11$ & $4.70 * 10-05$ & $4.78 * 10-05$ \\
\hline & $9.55 * 10-05$ & $8.55 * 10-06$ & $5.06 * 10-05$ & $4.35 * 10-05$ \\
\hline & $8.55 * 10-06$ & $1.48 * 10-06$ & $4.79 * 10-06$ & $3.56 * 10-06$ \\
\hline & 0.000185 & $4.33 * 10-06$ & $9.10 * 10-05$ & $9.06 * 10-05$ \\
\hline & 0.000248 & 0.000185 & 0.00022 & $3.20 * 10-05$ \\
\hline
\end{tabular}

\section{Conclusions}

Mathematical expression governing the dynamics of thin film flow of second grade fluid model is interpreted and analysed effectively by introducing a novel computing spline swarm
Table 4 Relative study on the basis of absolute errors of GA for spline 10 for cases $1-4$

\begin{tabular}{|c|c|c|c|c|}
\hline Case & Max & Min & Mean & Std \\
\hline \multirow[t]{10}{*}{1} & $6.45 * 10-07$ & $1.37 * 10-12$ & $4.02 * 10-07$ & $3.50 * 10-07$ \\
\hline & $7.51 * 10-07$ & $3.75 * 10-07$ & $5.90 * 10-07$ & $1.94 * 10-07$ \\
\hline & $3.75 * 10-07$ & $8.44 * 10-09$ & $2.13 * 10-07$ & $1.87 * 10-07$ \\
\hline & $7.59 * 10-07$ & $1.99 * 10-07$ & $4.05 * 10-07$ & $3.08 * 10-07$ \\
\hline & $7.59 * 10-07$ & $1.35 * 10-07$ & $3.55 * 10-07$ & $3.50 * 10-07$ \\
\hline & $1.21 * 10-06$ & $1.35 * 10-07$ & $5.88 * 10-07$ & $5.57 * 10-07$ \\
\hline & $1.21 * 10-06$ & $5.22 * 10-08$ & $4.85 * 10-07$ & $6.32 * 10-07$ \\
\hline & $1.38 * 10-06$ & $1.93 * 10-07$ & $6.43 * 10-07$ & $6.41 * 10-07$ \\
\hline & $1.38 * 10-06$ & $8.95 * 10-08$ & $6.76 * 10-07$ & $6.52 * 10-07$ \\
\hline & $1.03 * 10-06$ & $8.95 * 10-08$ & $5.25 * 10-07$ & $4.72 * 10-07$ \\
\hline \multirow[t]{10}{*}{2} & $6.26 * 10-06$ & $3.64 * 10-12$ & $3.13 * 10-06$ & $3.13 * 10-06$ \\
\hline & $6.26 * 10-06$ & $2.20 * 10-06$ & $4.19 * 10-06$ & $2.04 * 10-06$ \\
\hline & $4.12 * 10-06$ & $2.12 * 10-07$ & $2.77 * 10-06$ & $2.21 * 10-06$ \\
\hline & $4.90 * 10-06$ & $2.51 * 10-07$ & $3.04 * 10-06$ & $2.46 * 10-06$ \\
\hline & $4.90 * 10-06$ & $1.54 * 10-06$ & $2.72 * 10-06$ & $1.89 * 10-06$ \\
\hline & $6.15 * 10-06$ & $1.72 * 10-06$ & $3.41 * 10-06$ & $2.39 * 10-06$ \\
\hline & $6.15 * 10-06$ & $3.05 * 10-07$ & $3.20 * 10-06$ & $2.92 * 10-06$ \\
\hline & $7.91 * 10-06$ & $3.05 * 10-07$ & $3.99 * 10-06$ & $3.81 * 10-06$ \\
\hline & $7.91 * 10-06$ & $1.22 * 10-06$ & $4.51 * 10-06$ & $3.35 * 10-06$ \\
\hline & $8.11 * 10-06$ & $1.22 * 10-06$ & $4.64 * 10-06$ & $3.44 * 10-06$ \\
\hline \multirow[t]{10}{*}{3} & $1.59 * 10-05$ & $5.44 * 10-12$ & $8.00 * 10-06$ & $7.96 * 10-06$ \\
\hline & $1.59 * 10-05$ & $5.35 * 10-06$ & $9.01 * 10-06$ & $5.99 * 10-06$ \\
\hline & $9.71 * 10-06$ & $2.57 * 10-06$ & $6.02 * 10-06$ & $3.58 * 10-06$ \\
\hline & $1.15 * 10-05$ & $2.29 * 10-07$ & $7.15 * 10-06$ & $6.06 * 10-06$ \\
\hline & $1.15 * 10-05$ & $3.43 * 10-06$ & $6.24 * 10-06$ & $4.56 * 10-06$ \\
\hline & $1.65 * 10-05$ & $3.79 * 10-06$ & $8.58 * 10-06$ & $6.90 * 10-06$ \\
\hline & $1.65 * 10-05$ & $6.86 * 10-07$ & $8.69 * 10-06$ & $7.91 * 10-06$ \\
\hline & $1.97 * 10-05$ & $6.86 * 10-07$ & $1.03 * 10-05$ & $9.52 * 10-06$ \\
\hline & $1.97 * 10-05$ & $3.22 * 10-06$ & $1.14 * 10-05$ & $8.25 * 10-06$ \\
\hline & $2.13 * 10-05$ & $3.22 * 10-06$ & $1.23 * 10-05$ & $9.03 * 10-06$ \\
\hline \multirow[t]{10}{*}{4} & $3.18 * 10-05$ & $3.96 * 10-12$ & $1.58 * 10-05$ & $1.59 * 10-05$ \\
\hline & $3.18 * 10-05$ & $7.49 * 10-06$ & $1.71 * 10-05$ & $1.29 * 10-05$ \\
\hline & $2.11 * 10-05$ & $6.39 * 10-06$ & $1.17 * 10-05$ & $8.20 * 10-06$ \\
\hline & $2.11 * 10-05$ & $1.91 * 10-06$ & $1.36 * 10-05$ & $1.03 * 10-05$ \\
\hline & $1.77 * 10-05$ & $4.08 * 10-06$ & $1.08 * 10-05$ & $6.83 * 10-06$ \\
\hline & $2.76 * 10-05$ & $7.88 * 10-06$ & $1.53 * 10-05$ & $1.07 * 10-05$ \\
\hline & $2.76 * 10-05$ & $2.06 * 10-07$ & $1.37 * 10-05$ & $1.37 * 10-05$ \\
\hline & $3.45 * 10-05$ & $2.06 * 10-07$ & $1.72 * 10-05$ & $1.71 * 10-05$ \\
\hline & $3.45 * 10-05$ & $5.99 * 10-06$ & $2.05 * 10-05$ & $1.42 * 10-05$ \\
\hline & $3.82 * 10-05$ & $5.99 * 10-06$ & $2.22 * 10-05$ & $1.61 * 10-05$ \\
\hline
\end{tabular}

paradigm CSA-GA-SQP consisting of integration cubic spline approach, genetic algorithm, and sequential quadratic programming. The cubic spline approach with a length of 5,10 , and 15 is implemented as discretization to transform the differential equations of the TFF model into system of 
Table 5 Relative study on the basis of absolute errors of GA for spline 10 for cases $5-8$

\begin{tabular}{|c|c|c|c|c|}
\hline Case & Max & Min & Mean & Std \\
\hline \multirow[t]{10}{*}{5} & $4.42 * 10-05$ & $6.77 * 10-11$ & $2.20 * 10-05$ & $2.21 * 10-05$ \\
\hline & $4.42 * 10-05$ & $9.56 * 10-06$ & $2.41 * 10-05$ & $1.80 * 10-05$ \\
\hline & $3.05 * 10-05$ & $9.56 * 10-06$ & $1.67 * 10-05$ & $1.19 * 10-05$ \\
\hline & $3.05 * 10-05$ & $4.84 * 10-06$ & $1.92 * 10-05$ & $1.31 * 10-05$ \\
\hline & $2.24 * 10-05$ & $3.97 * 10-06$ & $1.36 * 10-05$ & $9.23 * 10-06$ \\
\hline & $3.71 * 10-05$ & $1.08 * 10-05$ & $2.07 * 10-05$ & $1.43 * 10-05$ \\
\hline & $3.71 * 10-05$ & $2.85 * 10-06$ & $2.01 * 10-05$ & $1.71 * 10-05$ \\
\hline & $5.10 * 10-05$ & $2.85 * 10-06$ & $2.68 * 10-05$ & $2.41 * 10-05$ \\
\hline & $5.10 * 10-05$ & $1.52 * 10-05$ & $3.31 * 10-05$ & $1.79 * 10-05$ \\
\hline & $5.67 * 10-05$ & $1.52 * 10-05$ & $3.60 * 10-05$ & $2.08 * 10-05$ \\
\hline \multirow[t]{10}{*}{6} & $4.80 * 10-05$ & $1.84 * 10-11$ & $2.38 * 10-05$ & $2.40 * 10-05$ \\
\hline & $4.80 * 10-05$ & $4.73 * 10-06$ & $2.49 * 10-05$ & $2.18 * 10-05$ \\
\hline & $3.63 * 10-05$ & $4.73 * 10-06$ & $1.87 * 10-05$ & $1.61 * 10-05$ \\
\hline & $3.63 * 10-05$ & $1.08 * 10-05$ & $2.09 * 10-05$ & $1.36 * 10-05$ \\
\hline & $1.89 * 10-05$ & $5.63 * 10-07$ & $1.17 * 10-05$ & $9.75 * 10-06$ \\
\hline & $3.31 * 10-05$ & $6.37 * 10-06$ & $1.95 * 10-05$ & $1.34 * 10-05$ \\
\hline & $3.31 * 10-05$ & $4.97 * 10-06$ & $1.93 * 10-05$ & $1.41 * 10-05$ \\
\hline & $5.51 * 10-05$ & $4.97 * 10-06$ & $2.99 * 10-05$ & $2.51 * 10-05$ \\
\hline & $5.51 * 10-05$ & $2.60 * 10-05$ & $4.06 * 10-05$ & $1.45 * 10-05$ \\
\hline & $6.59 * 10-05$ & $2.60 * 10-05$ & $4.62 * 10-05$ & $2.00 * 10-05$ \\
\hline \multirow[t]{10}{*}{7} & $3.16 * 10-05$ & $1.69 * 10-11$ & $1.57 * 10-05$ & $1.58 * 10-05$ \\
\hline & $3.16 * 10-05$ & $2.11 * 10-06$ & $1.63 * 10-05$ & $1.47 * 10-05$ \\
\hline & $2.50 * 10-05$ & $2.11 * 10-06$ & $1.28 * 10-05$ & $1.15 * 10-05$ \\
\hline & $2.50 * 10-05$ & $7.53 * 10-06$ & $1.41 * 10-05$ & $9.50 * 10-06$ \\
\hline & $9.78 * 10-06$ & $2.42 * 10-07$ & $6.47 * 10-06$ & $5.40 * 10-06$ \\
\hline & $2.82 * 10-05$ & $8.48 * 10-06$ & $1.54 * 10-05$ & $1.12 * 10-05$ \\
\hline & $2.82 * 10-05$ & $1.67 * 10-05$ & $2.23 * 10-05$ & $5.78 * 10-06$ \\
\hline & $5.50 * 10-05$ & $1.67 * 10-05$ & $3.55 * 10-05$ & $1.91 * 10-05$ \\
\hline & $5.50 * 10-05$ & $4.39 * 10-05$ & $4.94 * 10-05$ & $5.54 * 10-06$ \\
\hline & $7.06 * 10-05$ & $4.39 * 10-05$ & $5.76 * 10-05$ & $1.34 * 10-05$ \\
\hline \multirow[t]{10}{*}{8} & $2.02 * 10-05$ & $2.02 * 10-11$ & $1.00 * 10-05$ & $1.01 * 10-05$ \\
\hline & $2.02 * 10-05$ & $1.21 * 10-06$ & $1.09 * 10-05$ & $9.51 * 10-06$ \\
\hline & $1.68 * 10-05$ & $1.21 * 10-06$ & $8.92 * 10-06$ & $7.79 * 10-06$ \\
\hline & $1.68 * 10-05$ & $4.65 * 10-06$ & $9.26 * 10-06$ & $6.57 * 10-06$ \\
\hline & $4.82 * 10-06$ & $1.20 * 10-07$ & $3.20 * 10-06$ & $2.67 * 10-06$ \\
\hline & $2.11 * 10-05$ & $4.82 * 10-06$ & $1.11 * 10-05$ & $8.72 * 10-06$ \\
\hline & $2.11 * 10-05$ & $2.01 * 10-05$ & $2.05 * 10-05$ & $5.17 * 10-07$ \\
\hline & $5.11 * 10-05$ & $2.01 * 10-05$ & $3.55 * 10-05$ & $1.55 * 10-05$ \\
\hline & $5.25 * 10-05$ & $5.11 * 10-05$ & $5.20 * 10-05$ & $7.77 * 10-07$ \\
\hline & $7.19 * 10-05$ & $5.25 * 10-05$ & $6.26 * 10-05$ & $9.73 * 10-06$ \\
\hline
\end{tabular}

Table 6 Relative study on the basis of absolute errors of GA for spline 15 for cases $1-3$

\begin{tabular}{|c|c|c|c|c|}
\hline Case & Max & Min & Mean & Std \\
\hline \multirow[t]{15}{*}{1} & $6.36 * 10-07$ & $5.84 * 10-13$ & $2.96 * 10-07$ & $3.20 * 10-07$ \\
\hline & $6.36 * 10-07$ & $1.36 * 10-07$ & $3.89 * 10-07$ & $2.50 * 10-07$ \\
\hline & $5.34 * 10-07$ & $3.36 * 10-07$ & $4.21 * 10-07$ & $1.02 * 10-07$ \\
\hline & $3.36 * 10-07$ & $2.65 * 10-07$ & $3.01 * 10-07$ & $3.55 * 10-08$ \\
\hline & $6.19 * 10-07$ & $2.65 * 10-07$ & $4.00 * 10-07$ & $1.92 * 10-07$ \\
\hline & $6.19 * 10-07$ & $2.66 * 10-07$ & $4.12 * 10-07$ & $1.85 * 10-07$ \\
\hline & $3.70 * 10-07$ & $1.92 * 10-08$ & $2.47 * 10-07$ & $1.97 * 10-07$ \\
\hline & $5.46 * 10-07$ & $1.74 * 10-07$ & $3.63 * 10-07$ & $1.86 * 10-07$ \\
\hline & $1.86 * 10-07$ & $7.42 * 10-08$ & $1.45 * 10-07$ & $6.15 * 10-08$ \\
\hline & $8.39 * 10-07$ & $1.86 * 10-07$ & $4.57 * 10-07$ & $3.40 * 10-07$ \\
\hline & $8.39 * 10-07$ & $1.07 * 10-07$ & $4.61 * 10-07$ & $3.67 * 10-07$ \\
\hline & $7.54 * 10-07$ & $4.37 * 10-07$ & $6.05 * 10-07$ & $1.59 * 10-07$ \\
\hline & $7.54 * 10-07$ & $7.56 * 10-08$ & $4.18 * 10-07$ & $3.39 * 10-07$ \\
\hline & $1.56 * 10-07$ & $7.56 * 10-08$ & $1.29 * 10-07$ & $4.62 * 10-08$ \\
\hline & $5.80 * 10-07$ & $1.05 * 10-07$ & $2.80 * 10-07$ & $2.61 * 10-07$ \\
\hline \multirow[t]{15}{*}{2} & $3.42 * 10-06$ & $1.97 * 10-11$ & $1.51 * 10-06$ & $1.74 * 10-06$ \\
\hline & $3.42 * 10-06$ & $8.89 * 10-07$ & $1.93 * 10-06$ & $1.32 * 10-06$ \\
\hline & $2.07 * 10-06$ & $8.89 * 10-07$ & $1.36 * 10-06$ & $6.25 * 10-07$ \\
\hline & $2.07 * 10-06$ & $5.11 * 10-07$ & $1.49 * 10-06$ & $8.50 * 10-07$ \\
\hline & $1.88 * 10-06$ & $5.47 * 10-07$ & $1.24 * 10-06$ & $6.69 * 10-07$ \\
\hline & $2.31 * 10-06$ & $1.58 * 10-07$ & $1.26 * 10-06$ & $1.08 * 10-06$ \\
\hline & $2.31 * 10-06$ & $6.76 * 10-07$ & $1.38 * 10-06$ & $8.44 * 10-07$ \\
\hline & $2.28 * 10-06$ & $4.97 * 10-07$ & $1.31 * 10-06$ & $9.01 * 10-07$ \\
\hline & $2.28 * 10-06$ & $8.87 * 10-07$ & $1.35 * 10-06$ & $8.00 * 10-07$ \\
\hline & $2.60 * 10-06$ & $7.20 * 10-07$ & $1.40 * 10-06$ & $1.04 * 10-06$ \\
\hline & $2.60 * 10-06$ & $1.15 * 10-07$ & $1.47 * 10-06$ & $1.26 * 10-06$ \\
\hline & $3.73 * 10-06$ & $1.15 * 10-07$ & $1.68 * 10-06$ & $1.85 * 10-06$ \\
\hline & $3.73 * 10-06$ & $3.05 * 10-08$ & $1.79 * 10-06$ & $1.85 * 10-06$ \\
\hline & $3.29 * 10-06$ & $3.05 * 10-08$ & $1.82 * 10-06$ & $1.65 * 10-06$ \\
\hline & $3.29 * 10-06$ & $2.58 * 10-07$ & $1.80 * 10-06$ & $1.52 * 10-06$ \\
\hline \multirow[t]{15}{*}{3} & $7.79 * 10-06$ & $8.32 * 10-10$ & $3.98 * 10-06$ & $3.90 * 10-06$ \\
\hline & $1.19 * 10-05$ & $1.66 * 10-06$ & $7.12 * 10-06$ & $5.15 * 10-06$ \\
\hline & $6.04 * 10-06$ & $1.66 * 10-06$ & $3.23 * 10-06$ & $2.44 * 10-06$ \\
\hline & $6.04 * 10-06$ & $1.68 * 10-06$ & $3.74 * 10-06$ & $2.19 * 10-06$ \\
\hline & $4.94 * 10-06$ & $1.72 * 10-07$ & $2.86 * 10-06$ & $2.44 * 10-06$ \\
\hline & $5.31 * 10-06$ & $1.48 * 10-08$ & $3.42 * 10-06$ & $2.95 * 10-06$ \\
\hline & $5.31 * 10-06$ & $7.44 * 10-07$ & $2.98 * 10-06$ & $2.28 * 10-06$ \\
\hline & $6.40 * 10-06$ & $2.26 * 10-06$ & $3.85 * 10-06$ & $2.23 * 10-06$ \\
\hline & $6.40 * 10-06$ & $7.89 * 10-07$ & $3.29 * 10-06$ & $2.85 * 10-06$ \\
\hline & $8.14 * 10-06$ & $7.88 * 10-07$ & $4.01 * 10-06$ & $3.76 * 10-06$ \\
\hline & $8.14 * 10-06$ & $4.53 * 10-07$ & $4.23 * 10-06$ & $3.84 * 10-06$ \\
\hline & $8.76 * 10-06$ & $4.54 * 10-07$ & $4.70 * 10-06$ & $4.16 * 10-06$ \\
\hline & $8.76 * 10-06$ & $6.70 * 10-07$ & $4.70 * 10-06$ & $4.05 * 10-06$ \\
\hline & $9.01 * 10-06$ & $6.71 * 10-07$ & $4.91 * 10-06$ & $4.17 * 10-06$ \\
\hline & $9.01 * 10-06$ & $1.49 * 10-06$ & $5.12 * 10-06$ & $3.77 * 10-06$ \\
\hline
\end{tabular}


Table 7 Relative study on the basis of absolute errors of GA for spline 15 for cases 4-6

\begin{tabular}{|c|c|c|c|c|}
\hline Case & Max & Min & Mean & Std \\
\hline \multirow[t]{15}{*}{4} & $1.32 * 10-05$ & $2.70 * 10-11$ & $6.68 * 10-06$ & $6.59 * 10-06$ \\
\hline & $1.32 * 10-05$ & $2.27 * 10-06$ & $6.95 * 10-06$ & $5.61 * 10-06$ \\
\hline & $1.07 * 10-05$ & $2.27 * 10-06$ & $5.47 * 10-06$ & $4.59 * 10-06$ \\
\hline & $1.07 * 10-05$ & $2.88 * 10-06$ & $6.26 * 10-06$ & $4.03 * 10-06$ \\
\hline & $7.10 * 10-06$ & $6.24 * 10-07$ & $4.30 * 10-06$ & $3.33 * 10-06$ \\
\hline & $8.13 * 10-06$ & $1.78 * 10-08$ & $5.08 * 10-06$ & $4.42 * 10-06$ \\
\hline & $8.13 * 10-06$ & $2.57 * 10-06$ & $4.82 * 10-06$ & $2.93 * 10-06$ \\
\hline & $1.04 * 10-05$ & $3.19 * 10-06$ & $5.79 * 10-06$ & $4.03 * 10-06$ \\
\hline & $1.04 * 10-05$ & $1.26 * 10-07$ & $5.18 * 10-06$ & $5.16 * 10-06$ \\
\hline & $1.35 * 10-05$ & $1.26 * 10-07$ & $6.65 * 10-06$ & $6.67 * 10-06$ \\
\hline & $1.35 * 10-05$ & $2.37 * 10-06$ & $8.02 * 10-06$ & $5.55 * 10-06$ \\
\hline & $1.52 * 10-05$ & $2.37 * 10-06$ & $8.83 * 10-06$ & $6.42 * 10-06$ \\
\hline & $1.52 * 10-05$ & $3.77 * 10-06$ & $9.61 * 10-06$ & $5.73 * 10-06$ \\
\hline & $1.65 * 10-05$ & $3.77 * 10-06$ & $1.02 * 10-05$ & $6.38 * 10-06$ \\
\hline & $1.65 * 10-05$ & $4.95 * 10-06$ & $1.08 * 10-05$ & $5.79 * 10-06$ \\
\hline \multirow[t]{15}{*}{5} & $1.49 * 10-05$ & $5.54 * 10-12$ & $7.51 * 10-06$ & $7.47 * 10-06$ \\
\hline & $1.49 * 10-05$ & $2.66 * 10-06$ & $7.92 * 10-06$ & $6.33 * 10-06$ \\
\hline & $1.11 * 10-05$ & $2.66 * 10-06$ & $5.87 * 10-06$ & $4.58 * 10-06$ \\
\hline & $1.11 * 10-05$ & $2.60 * 10-06$ & $6.75 * 10-06$ & $4.26 * 10-06$ \\
\hline & $7.43 * 10-06$ & $1.96 * 10-07$ & $4.72 * 10-06$ & $3.94 * 10-06$ \\
\hline & $1.06 * 10-05$ & $1.20 * 10-06$ & $6.40 * 10-06$ & $4.76 * 10-06$ \\
\hline & $1.06 * 10-05$ & $2.17 * 10-06$ & $5.67 * 10-06$ & $4.36 * 10-06$ \\
\hline & $1.42 * 10-05$ & $2.17 * 10-06$ & $7.37 * 10-06$ & $6.19 * 10-06$ \\
\hline & $1.42 * 10-05$ & $2.78 * 10-06$ & $8.51 * 10-06$ & $5.72 * 10-06$ \\
\hline & $1.97 * 10-05$ & $2.78 * 10-06$ & $1.13 * 10-05$ & $8.45 * 10-06$ \\
\hline & $1.97 * 10-05$ & $7.70 * 10-06$ & $1.35 * 10-05$ & $6.00 * 10-06$ \\
\hline & $2.33 * 10-05$ & $7.70 * 10-06$ & $1.56 * 10-05$ & $7.83 * 10-06$ \\
\hline & $2.33 * 10-05$ & $1.05 * 10-05$ & $1.69 * 10-05$ & $6.40 * 10-06$ \\
\hline & $2.51 * 10-05$ & $1.05 * 10-05$ & $1.80 * 10-05$ & $7.27 * 10-06$ \\
\hline & $2.51 * 10-05$ & $1.25 * 10-05$ & $1.90 * 10-05$ & $6.31 * 10-06$ \\
\hline \multirow[t]{15}{*}{6} & $1.88 * 10-05$ & $3.25 * 10-10$ & $9.49 * 10-06$ & $9.41 * 10-06$ \\
\hline & $1.88 * 10-05$ & $1.59 * 10-06$ & $9.91 * 10-06$ & $8.63 * 10-06$ \\
\hline & $1.65 * 10-05$ & $1.59 * 10-06$ & $8.30 * 10-06$ & $7.56 * 10-06$ \\
\hline & $1.65 * 10-05$ & $3.85 * 10-06$ & $8.91 * 10-06$ & $6.69 * 10-06$ \\
\hline & $1.20 * 10-05$ & $3.85 * 10-06$ & $6.60 * 10-06$ & $4.68 * 10-06$ \\
\hline & $1.20 * 10-05$ & $2.60 * 10-06$ & $7.22 * 10-06$ & $4.71 * 10-06$ \\
\hline & $7.44 * 10-06$ & $3.22 * 10-07$ & $4.94 * 10-06$ & $4.01 * 10-06$ \\
\hline & $1.26 * 10-05$ & $2.20 * 10-06$ & $7.41 * 10-06$ & $5.19 * 10-06$ \\
\hline & $1.26 * 10-05$ & $1.07 * 10-06$ & $6.44 * 10-06$ & $5.80 * 10-06$ \\
\hline & $1.83 * 10-05$ & $1.07 * 10-06$ & $9.43 * 10-06$ & $8.62 * 10-06$ \\
\hline & $1.83 * 10-05$ & $6.29 * 10-06$ & $1.23 * 10-05$ & $6.00 * 10-06$ \\
\hline & $2.48 * 10-05$ & $6.29 * 10-06$ & $1.58 * 10-05$ & $9.28 * 10-06$ \\
\hline & $2.48 * 10-05$ & $1.25 * 10-05$ & $1.88 * 10-05$ & $6.19 * 10-06$ \\
\hline & $2.90 * 10-05$ & $1.25 * 10-05$ & $2.08 * 10-05$ & $8.24 * 10-06$ \\
\hline & $2.90 * 10-05$ & $1.50 * 10-05$ & $2.21 * 10-05$ & $6.98 * 10-06$ \\
\hline
\end{tabular}

Table 8 Relative study on the basis of absolute errors of GA for spline 10 for cases 7-8

\begin{tabular}{lllll}
\hline Case & Max & Min & Mean & Std \\
\hline 7 & $1.88 * 10-05$ & $3.73 * 10-11$ & $9.28 * 10-06$ & $9.42 * 10-06$ \\
$1.88 * 10-05$ & $6.24 * 10-07$ & $9.66 * 10-06$ & $9.10 * 10-06$ \\
$1.69 * 10-05$ & $6.24 * 10-07$ & $8.52 * 10-06$ & $8.15 * 10-06$ \\
$1.69 * 10-05$ & $1.80 * 10-06$ & $9.02 * 10-06$ & $7.57 * 10-06$ \\
$1.40 * 10-05$ & $1.80 * 10-06$ & $7.53 * 10-06$ & $6.15 * 10-06$ \\
$1.40 * 10-05$ & $4.08 * 10-06$ & $7.77 * 10-06$ & $5.46 * 10-06$ \\
$1.00 * 10-05$ & $2.49 * 10-06$ & $5.53 * 10-06$ & $3.97 * 10-06$ \\
$1.00 * 10-05$ & $7.09 * 10-07$ & $6.44 * 10-06$ & $5.01 * 10-06$ \\
$8.59 * 10-06$ & $2.42 * 10-06$ & $4.81 * 10-06$ & $3.31 * 10-06$ \\
$1.53 * 10-05$ & $2.42 * 10-06$ & $7.83 * 10-06$ & $6.68 * 10-06$ \\
$1.53 * 10-05$ & $5.86 * 10-06$ & $1.07 * 10-05$ & $4.73 * 10-06$ \\
$2.41 * 10-05$ & $5.86 * 10-06$ & $1.49 * 10-05$ & $9.11 * 10-06$ \\
$2.41 * 10-05$ & $1.40 * 10-05$ & $1.89 * 10-05$ & $5.03 * 10-06$ \\
$3.01 * 10-05$ & $1.40 * 10-05$ & $2.20 * 10-05$ & $8.05 * 10-06$ \\
$3.01 * 10-05$ & $1.73 * 10-05$ & $2.37 * 10-05$ & $6.42 * 10-06$ \\
$1.18 * 10-05$ & $1.09 * 10-10$ & $5.77 * 10-06$ & $5.89 * 10-06$ \\
$1.18 * 10-05$ & $2.77 * 10-07$ & $5.95 * 10-06$ & $5.75 * 10-06$ \\
$1.17 * 10-05$ & $2.77 * 10-07$ & $6.08 * 10-06$ & $5.73 * 10-06$ \\
$1.17 * 10-05$ & $3.45 * 10-07$ & $5.99 * 10-06$ & $5.69 * 10-06$ \\
$9.86 * 10-06$ & $3.45 * 10-07$ & $4.79 * 10-06$ & $4.79 * 10-06$ \\
$9.86 * 10-06$ & $2.04 * 10-06$ & $5.33 * 10-06$ & $4.05 * 10-06$ \\
$6.00 * 10-06$ & $2.04 * 10-06$ & $3.37 * 10-06$ & $2.28 * 10-06$ \\
$6.20 * 10-06$ & $4.48 * 10-07$ & $4.22 * 10-06$ & $3.26 * 10-06$ \\
$6.20 * 10-06$ & $4.07 * 10-07$ & $3.39 * 10-06$ & $2.90 * 10-06$ \\
$1.33 * 10-05$ & $4.07 * 10-07$ & $6.70 * 10-06$ & $6.45 * 10-06$ \\
$1.33 * 10-05$ & $9.56 * 10-06$ & $1.14 * 10-05$ & $1.87 * 10-06$ \\
$2.26 * 10-05$ & $9.56 * 10-06$ & $1.60 * 10-05$ & $6.52 * 10-06$ \\
$2.26 * 10-05$ & $1.94 * 10-05$ & $2.08 * 10-05$ & $1.64 * 10-06$ \\
$3.01 * 10-05$ & $1.94 * 10-05$ & $2.46 * 10-05$ & $5.35 * 10-06$ \\
$3.01 * 10-05$ & $2.34 * 10-05$ & $2.69 * 10-05$ & $3.34 * 10-06$ \\
8 & & &
\end{tabular}

Table 9 Cases description for thin film flow model

\begin{tabular}{lll}
\hline Index & $\beta$ & $\mathrm{n}$ \\
\hline 1 & $5.00 * 10-01$ & $1.00 * 10-01$ \\
2 & $5.00 * 10-01$ & $2.00 * 10-01$ \\
3 & $5.00 * 10-01$ & $3.00 * 10-01$ \\
4 & $5.00 * 10-01$ & $4.00 * 10-01$ \\
5 & $5.00 * 10-01$ & $5.00 * 10-01$ \\
6 & $1.00 \mathrm{E}+00$ & $5.00 * 10-01$ \\
7 & $1.50 \mathrm{E}+00$ & $5.00 * 10-01$ \\
8 & $2.00 \mathrm{E}+00$ & $5.00 * 10-01$ \\
\hline
\end{tabular}


nonlinear equations. The approximation in mean squared error sense is exploited to represent the objective function of TFF model, and combined strength of GAs and SQP is utilized for optimization. The designed spline-evolutionary computing paradigm, CSA-GA-SQP, is evaluated for different scenarios of TFF model by variation of second grade, $\beta$, and magnetic, $\mathrm{m}$, parameters. Results with reasonable accuracy level certified the performance of CSA-GA-SQP solver as an efficient alternative, reliable, and stable platform for the variants of nonlinear TFF system.

In the future, the design computing spline swarm paradigm CSA-GA-SQP is a promising alternative numerical solver to be implemented for the solution of stiff nonlinear systems representing the complex scenarios of computational fluid dynamics problems [63-70].

\section{References}

1. Siddiqui, A.M.; Mahmood, R.; Ghori, Q.K.: Thin film flow of a third grade fluid on a moving belt by Hes homotopy perturbation method. Int. J. Nonlinear Sci. Numer. Simul. 7(1), 7-14 (2006)

2. Ahmad, I.; Mukhtar, B.; Kutlu, K.; Ahmad, F.: A simple neuroheuristic computational intelligence algorithm for thin film flow equation arising in physical models. In: Proceedings of the 2017 16th IEEE International Conference on Machine Learning and Applications (ICMLA) (pp. 556-561). IEEE (2017)

3. Sakiadis, B.C.: Boundary-layer behavior on continuous solid surfaces. AICh Eng. J. 7(1461), 26-28

4. Chen, C.H.: Mixed convection cooling of a heated continuously stretching surface. Heat Mass Transfer 36, 79-86 (2000)

5. Zhang, R.; Li, X.: Non-Newtonian effects on lubricant thin film flows. J. Eng. Math. 51, 1-13 (2005)

6. Elahi, R.; Riaz, A.: Analytical solution for MHD flow in a third grade fluid with variable viscosity. Math. Comput. Model 52, 17831793 (2010)

7. Agrawal, V.P.; Denman, N.H.: Weighted linearization technique for period approximation in large amplitude nonlinear oscillations. J. Sound Vibr. 99, 463-473 (1985)

8. Hagedorn, P.: Nonlinear Oscillations. Clarendon Press, Oxford (1981)

9. Adomian, G.: A review of decomposition method in applied mathematics. J. Math. Anal. Appl. 135, 501-544 (1995)

10. Wu, Y.Y.; Liao, S.J.; Zhao, X.Z.: Some notes on the general boundary element method for highly nonlinear problems. Commun. Nonlinear Sci. Num. Simul. 10, 725-735 (2005)

11. Marinca, V.; Herisanu, N.: Nonlinear Dynamical Systems in Engineering, Some approximate Approaches. Springer, Berlin, Heidelberg (2011)

12. Marinca, V.; Herisanu, N.: The Optimal Homotopy Asymptotic Method. Engineering Applications. Springer, Chaim (2015)

13. Siddiqui, A.M.; Mahmood, R.; Ghori, Q.K.: Homotopy perturbation method for thin film flow of a fourth grade fluid. Phys. Lett. A 352, 404-410 (2006)

14. Sajid, M.; Hayat, F.; Asghar, S.: On the analytic solution of the steady flow of a fourth grade fluid. Phys. Lett. 355, 18-26 (2006)

15. Hayat, T.; Sajid, M.: An analytic solution for thin film flow of a fourth grade fluid down a vertical cylinder. Phys. Lett. A 361, 316-322 (2007)
16. Mahmood, F.: Comparison of optimal bomotopy asymptotic method and homotopy perturbation method for strongly non-linear equation. J. Assoc. Arab Univ. Basic Appl. Sci. 16, 21-26 (2014)

17. Marinca, V.; Herisanu, N.; Nemes, I.: Optimal bomotopy asymptotic method with application to thin flow. Cent. Eur. J. Phys. 16, 648-653 (2008)

18. Marinca, V.; Herisanu, N.: An optimal homotopy perturbation approach to thin film flow of a fourth grade fluid. AIP Conf. Proc. 1479, 2383-2386 (2012)

19. Marinca, V.; Ene, R.D.; Marinca, B.; Negrea, R.: Different approximation to the solution of upper-convected Maxwell fluid on a porous stretching plate. Abst. Appl. Anal. Article ID 139314 (2014)

20. Ancey, C.: Plasticity and geophysical flows: a review. J. NonNewton. Fluid Mech. 142, 4-35 (2007)

21. Rizwana, R.; Hussain, A.; Nadeem, S.: Mix convection nonboundary layer flow of unsteady MHD oblique stagnation point flow of nanofluid. Int. Commun. Heat Mass Transf. 124, 105285 (2021)

22. Yasin, A.; Ullah, N.; Saleem, S.; Nadeem, S.; Al-Zubaidi, A.: Impact of uniform and non-uniform heated rods on free convective flow inside a porous enclosure: finite element analysis. Phys. Scripta (2021)

23. Awan, A.U.; Aziz, M.; Ullah, N.; Nadeem, S.; Abro, K.A.: Thermal analysis of oblique stagnation point flow with slippage on secondorder fluid. J. Therm. Anal. Calorim. 1-13 (2021)

24. Ahmad, S.; Nadeem, S.; Khan, M.N.: Mixed convection hybridized micropolar nanofluid with triple stratification and CattaneoChristov heat flux model. Phys. Scripta (2021)

25. Yasin, A.; Ullah, N.; Nadeem, S.; Saleem, S.: Finite element simulation for free convective flow in an adiabatic enclosure: study of Lorentz forces and partially thermal walls. Case Stud. Therm. Eng. 100981 (2021)

26. Hussain, A.; Rehman, A.; Nadeem, S.; Malik, M.Y.; Issakhov, A.; Sarwar, L.; Hussain, S.: A combined convection Carreau-Yasuda nanofluid model over a convective heated surface near a stagnation point: a numerical study. Math. Prob. Eng. (2021)

27. Abbas, N.; Nadeem, S.; Issakhov, A.: Transportation of modified nanofluid flow with time dependent viscosity over a Riga plate: exponentially stretching. Ain Shams Eng. J. (2021)

28. Khan, M.N.; Ahmad, S.; Nadeem, S.: Flow and heat transfer investigation of bio-convective hybrid nanofluid with triple stratification effects. Phys. Scr. 96(6), 065210 (2021)

29. Sabir, Z.; et al.: Neuro-swarms intelligent computing using Gudermannian kernel for solving a class of second order Lane-Emden singular nonlinear model. AIMS Math. 6(3), 2468-2485 (2021)

30. Almalki, M.M.; et al.: A Levenberg-Marquardt backpropagation neural network for the numerical treatment of squeezing flow with heat transfer model. IEEE Access (2020)

31. Ahmad, I.; Raja, M.A.Z.; Bilal, M.; Ashraf, F.: Bio-inspired computational heuristics to study Lane-Emden systems arising in astrophysics model. SpringerPlus 5(1), 1866 (2016)

32. Ahmad, I.; Ahmad, F.; Raja, M.A.Z.; Ilyas, H.; Anwar, N.; Azad,Z.: Intelligent computing to solve fifth-order boundary value problem arising in induction motor models. Neural Comput. Appl. 29(7), 449-466 (2018)

33. Umar, M.; et al.: A stochastic numerical computing heuristic of SIR nonlinear model based on dengue fever. Results Phys. 19, 103585 (2020)

34. Raja, M.A.Z.; Manzar, M.A.; Samar, R.: An efficient computational intelligence approach for solving fractional order Riccati equations using ANN and SQP. Appl. Math. Model. 39(10-11), 3075-3093 (2015)

35. Ahmad, I.; Ilyas, H.; Urooj, A.; Aslam, M.S.; Shoaib, M.; Raja, M.A.Z.: Novel applications of intelligent computing paradigms for the analysis of nonlinear reactive transport model of the fluid in 
soft tissues and microvessels. Neural Comput. Appl. 31(12), 90419059 (2019)

36. Sabir, Z.; et al.: FMNEICS: fractional Meyer neuro-evolutionbased intelligent computing solver for doubly singular multifractional order Lane-Emden system. Comput. Appl. Math. 39(4), $1-18(2020)$

37. Ahmad, I.; Zahid, H.; Ahmad, F.; Raja, M.A.Z.; Baleanu, D.: Design of computational intelligent procedure for thermal analysis of porous fin model. Chin. J. Phys. 59, 641-655 (2019)

38. Jadoon, I.; et al.: Integrated meta-heuristics finite difference method for the dynamics of nonlinear unipolar electrohydrodynamic pump flow model. Appl. Soft Comput. 97, 106791 (2020)

39. Raja, M.A.Z.; Khan, J.A.; Qureshi, I.M.: A new stochastic approach for solution of Riccati differential equation of fractional order. Ann. Math. Artif. Intell. 60(3-4), 229-250 (2010)

40. Raja, M.A.Z.; Shah, F.H.; Tariq, M.; Ahmad, I.: Design of artificial neural network models optimized with sequential quadratic programming to study the dynamics of nonlinear Troeschs problem arising in plasma physics. Neural Comput. Appl. 29(6), 83-109 (2018)

41. Shah, Z.; et al.: Design of neural network based intelligent computing for neumerical treatment of unsteady 3D flow of Eyring-Powell magneto-nanofluidic model. J. Mater. Res. Technol. 9(6), 1437214387 (2020)

42. Bukhari, A.H.; Sulaiman, M.; Raja, M.A.Z.; Islam, S.; Shoaib, M.; Kumam, P.: Design of a hybrid NAR-RBFs neural network for nonlinear dusty plasma system. Alex. Eng. J. 59(5), 3325-3345 (2020)

43. Sabir, Z.; et al.: A neuro-swarming intelligence-based computing for second order singular periodic non-linear boundary value problems. Front. Phys. 8, 224 (2020)

44. Ilyas, H.; Ahmad, I.; Raja, M.A.Z.; Shoaib, M.: A novel design of Gaussian WaveNets for rotational hybrid nanofluidic flow over a stretching sheet involving thermal radiation. Int. Commun. Heat Mass Transfer 123, 105196 (2021)

45. Ilyas, H.; Ahmad, I.; Raja, M.A.Z.; Tahir, M.B.; Shoaib, M.: Intelligent networks for crosswise stream nanofluidic model with $\mathrm{Cu}-\mathrm{H}_{2} \mathrm{O}$ over porous stretching medium. Int. J. Hydrogen Energy (2021)

46. Ilyas, H.; Ahmad, I.; Raja, M.A.Z.; Tahir, M.B.; Shoaib, M.: Intelligent computing for the dynamics of fluidic system of electrically conducting $\mathrm{Ag} / \mathrm{Cu}$ nanoparticles with mixed convection for hydrogen possessions. Int. J. Hydrogen Energy 46(7), 4947-4980 (2021)

47. Cheema, T.N.; Raja, M.A.Z.; Ahmad, I.; Naz, S.; Ilyas, H.; Shoaib, M.: Intelligent computing with Levenberg-Marquardt artificial neural networks for nonlinear system of COVID-19 epidemic model for future generation disease control. Eur. Phys. J. Plus 135(11), 1-35 (2020)

48. Ahmad, I.; Raja, M.A.Z.; Ramos, H.; Bilal, M.; Shoaib, M.: Integrated neuro-evolution-based computing solver for dynamics of nonlinear corneal shape model numerically. Neural Comput. Appl. $1-17(2020)$

49. Tirmizi, I.A.; Haq, F.: Quartic non-polynomial splines approach to the solution of a system of second-order boundary-value problems. Int. J. High Perform. Comput. Appl. 21(1), 42-49 (2007)

50. Tung, M. M. S.: Spline approximations for systems of ordinary differential equations (Doctoral dissertation) (2013)

51. Al-Towaiq, M.; Alayed, O.: An efficient algorithm based on the cubic spline for the solution of Bratu-type equation. J. Interdiscip. Math. 17(5-6), 471-484 (2014)

52. Karim, S.A.A.; Rosli, M.A.M.; Mustafa, M.I.M.: Cubic spline interpolation for petroleum engineering data. Appl. Math. Sci. 8(102), 5083-5098 (2014)
53. Akram, G.: Solution of the system of fifth order boundary value problem using sextic spline. J. Egypt. Math. Soc. 23(2), 406-409 (2015)

54. He, S.; Fang, S.; Liu, X.; Zhang, W.; Xie, W.; Zhang, H.; Pei, D.: Investigation of a genetic algorithm based cubic spline smoothing for baseline correction of Raman spectra. Chemometr. Intell. Lab. Syst. 152, 1-9 (2016)

55. Ahmad, N.; Deeba, K.F.: The study of new approaches in cubic spline interpolation for auto mobile data. J. Sci. Arts 17(3), 401406 (2017)

56. Mirzaee, F.; Alipour, S.: Cubic B-spline approximation for linear stochastic integro-differential equation of fractional order. J. Comput. Appl. Math. 366, 112440 (2020)

57. Ahmad, S.U.I.; Faisal, F.; Shoaib, M.; Raja, M.A.Z.: A new heuristic computational solver for nonlinear singular Thomas-Fermi system using evolutionary optimized cubic splines. Eur. Phys. J. Plus 135, 1-29 (2020)

58. Raja, M. A. Z.; Khan, J. A.; Ahmad, S. U. I.; Qureshi, I. M.: A new stochastic technique for Painlevequation-I using neural network optimized with swarm intelligence. Comput. Intell. Neurosci. (2012)

59. Siddiqui, A.M.; Mahmood, R.; Ghori, Q.K.: Homotopy perturbation method for thin film flow of a third grade fluid down an inclined plane. Chaos Solitons Fractals 35(1), 140-147 (2008)

60. Zadeh, L.A.: Soft computing and fuzzy logic. Software 11(6), 48 56 (1994)

61. Holland, J.H.: Adaptation in natural and artificial systems: an introductory analysis with applications to biology, control, and artificial intelligence. MIT press, Cambridge (1992)

62. John, H.: Holland. genetic algorithms. Sci. Am. 267(1), 44-50 (1992)

63. Khan, I.; et al.: Design of Neural Network With LevenbergMarquardt and Bayesian regularization backpropagation for solving pantograph delay differential equations. IEEE Access 8, 137918-137933 (2020)

64. Esfe, M.H.; Motallebi, S.M.; Bahiraei, M.: Employing response surface methodology and neural network to accurately model thermal conductivity of $\mathrm{TiO}_{2}$-water nanofluid using experimental data. Chin. J. Phys. (2020)

65. Waini, I.; Ishak, A.; Pop, I.: Hybrid nanofluid flow on a shrinking cylinder with prescribed surface heat flux. Int. J. Numer. Methods Heat Fluid Flow (2020)

66. Khan, Y.: A variational approach for novel solitary solutions of FitzHugh-Nagumo equation arising in the nonlinear reactiondiffusion equation. Int. J. Numer. Methods Heat Fluid Flow (2020)

67. Akram, J.; Akbar, N.S.; Tripathi, D.: Numerical simulation of electrokinetically driven peristaltic pumping of silver-water nanofluids in an asymmetric microchannel. Chin. J. Phys. 68, 745-763 (2020)

68. Shahzadi, I.; Kausar, N.: Doubly stratified non-Newtonian nanofluid flow featuring nonlinear convection. Int. J. Numer. Methods Heat Fluid Flow (2020)

69. Zahid, U.M.; Akbar, Y.; Abbasi, F.M.: Entropy generation analysis for peristaltically driven flow of hybrid nanofluid. Chin. J. Phys. 67, 330-348 (2020)

70. Salehi, M.; Pourmahmoud, N.; Hassanzadeh, A.; Hoseinzadeh, S.; Heyns, P.S.: Computational fluid dynamics analysis of the effect of throat diameter on the fluid flow and performance of ejector. Int. J. Numer. Methods Heat Fluid Flow (2020) 